\title{
Synthesis, Characterization, Crystal Structure and Quantum Chemical Calculations of 2-oxo-2H-chromen-3-yl Acetate
}

\author{
Akoun Abou ${ }^{1, *}$, Siaka Sosso ${ }^{2}$, Affiba Florance Kouassi ${ }^{3}$, Thouakesseh Jeremie Zoueu ${ }^{1}$, \\ Abdoulaye Djande ${ }^{2}$, Olivier Ouari ${ }^{4}$ \\ ${ }^{1}$ Department of Training and Research in Electrical and Electronic Engineering, Research Team: Instrumentation, Image and Spectroscopy, \\ Félix Houphouët-Boigny National Polytechnic Institute, Yamoussoukro, Côte d'Ivoire \\ ${ }^{2}$ Department of Chemistry, Laboratory of Molecular Chemistry and Materials, Research Team: Organic Chemistry and Phytochemistry, \\ University Joseph KI-ZERBO, Ouagadougou, Burkina Faso \\ ${ }^{3}$ Department of Physics, Laboratory of Fundamental and Applied Physics, University of Abobo Adjamé—-Nangui Abrogoua, Abidjan, Côte \\ d'Ivoire \\ ${ }^{4}$ Department of Chemistry, Radical Chemistry Institute, Research Team SREP, Aix-Marseille University, Marseille, France
}

\section{Email address:}

abouakoun@gmail.com (A. Abou), sossosiaka@yahoo.fr (S. Sosso), akouassi859@yahoo.com (A. F. Kouassi), jzoueu@gmail.com (T. J. Zoueu), djandeabdou@yahoo.fr (A. Djande), olivier.ouari@univ-amu.fr (O. Ouari)

${ }^{*}$ Corresponding author

\section{To cite this article:}

Akoun Abou, Siaka Sosso, Affiba Florance Kouassi, Thouakesseh Jeremie Zoueu, Abdoulaye Djande, Olivier Ouari. Synthesis, Characterization, Crystal Structure and Quantum Chemical Calculations of 2-oxo-2H-chromen-3-yl Acetate. Science Journal of Chemistry. Vol. 9, No. 2, 2021, pp. 29-44. doi: 10.11648/j.sjc.20210902.11

Received: March 14, 2021; Accepted: April 6, 2021; Published: April 16, 2021

\begin{abstract}
This paper is focused on a combined experimental and theoretical study of 3-substituted coumarin derivative, the 2-oxo-2H-chromen-3-yl acetate (I). The compound was synthesized by reacting chroman-2,3-dione and acetic anhydride in dried diethyl ether in the presence of dried pyridine and crystallized in the orthorhombic crystal system with Pbca space group. The lattice parameters of the structure are $a=14.6770$ (1), $b=7.1079$ (1), $c=17.6767$ (2) $\AA, \alpha=\beta=\gamma=90^{\circ}$ with 8 molecules per unit cell $(Z=8)$. The compound has been characterized structurally by Spectroscopy utilizing ${ }^{1} \mathrm{H} N \mathrm{NR},{ }^{13} \mathrm{C}$ NMR and IR techniques and by crystallography using the X-Ray diffraction (XRD) analysis. In the crystallographic study, the positions of the atoms were determined by direct methods and refined to a final $\mathrm{R}$ value of 0.038 for 1768 independent reflections. The stabilization of the structure is provided by intermolecular $\mathrm{C}-\mathrm{H} \cdots \mathrm{O}$ hydrogen bonds extending along [010] direction. Likewise, the presence and nature of intermolecular contacts are determined by the 3-D molecular Hirshfeld surface and 2-D fingerprint plot analysis which indicate the main contributions to the Hirshfeld surface, $38.7 \%$ for $\mathrm{O} \cdots \mathrm{H}$ and $28.7 \%$ for $\mathrm{H} \cdot \mathrm{H}$. Moreover, the molecular geometry of (I) was as well minimized utilizing density functional theory (DFT/RB3LYP), the frequency calculations with RB3LYP method, the basic ab initio model i.e the restricted Hartree-Fock (RHF) and the exchange component of Perdew and Wang's 1991 functional B3PW91 methods with the 6-311 ${ }^{++} \mathrm{G}(\mathrm{d}, \mathrm{p})$ basis set in ground state. The derived structural parameters highlight very good correlation with the crystallographic results. Frontier molecular orbitals (HOMO-LUMO), their energy gap, the non-linear optical effects (NLO) and related reactive parameters were also computed to better apprehend the properties of the molecule.
\end{abstract}

Keywords: 3-substituted Coumarin Derivative, C-H...O Hydrogen Bonds, Hirshfeld Surface Analysis, Quantum Chemical Computations, Crystal Structure 


\section{Introduction}

Coumarin derivatives are of huge interest as useful materials and have attracted more and more attention nowadays because of their widespread biological properties covering anti-HIV [1, 2], anti-coagulant [3], anti-oxidant [4], anti-tumor [5], antibacterial [6], and anti-inflammatory [7].

Given their importance and as an extension of our work on the analysis of the crystal structure of coumarin derivatives [8-9], we report here the synthesis, the crystal structure, the geometry optimization and the Hirshfeld surface analysis of the title compound (I).

\section{Experimental and Theoretical Methods}

\subsection{Synthesis}

In a $100 \mathrm{ml}$ round-necked flask topped with a water condenser were introduced successively: dried diethyl ether $(25 \mathrm{~mL})$, acetic anhydride $(0.65 \mathrm{~mL} ; 6.17 \mathrm{mmol})$ and dried pyridine (2.35 mL; 4.7 molar equivalents). While stirring strongly, chroman-2,3-dione (1g; $6.17 \mathrm{mmol}$ ) was added in small portions over $30 \mathrm{~min}$. The reaction mixture was left under agitation at room temperature for $3 \mathrm{~h}$. The mixture was then poured in a separating funnel containing $40 \mathrm{~mL}$ of chloroform and washed with diluted hydrochloric acid solution until the $\mathrm{pH}$ was $2-3$. The organic phase was extracted, washed with water to neutrality, dried over $\mathrm{MgSO}_{4}$ and the solvent removed. The resulting precipitate was filtered off with suction, washed with petroleum ether and recrystallized from chloroform to obtain yellow laminar crystals of the title compound (I): yield 72\%; M.pt. 371-373 K.

\subsection{Electrospray Ionisation Mass Spectrum}

The analyses were carried out with a 3200 QTRAP spectrometer (Applied Biosystems SCIEX) equipped with a pneumatically assisted air pressure ionization (API) source for ESI-MS ${ }^{+}$experiment. The sample in solution was ionized in the following conditions: electrospray tension (ISV): 5500 $\mathrm{V}$; orifice tension (OR): $20 \mathrm{~V}$; nebulizing gas pressure (air): 10 psi. The mass spectrum (figure 2) was obtained with a quadrupole analyzer.

\subsection{IR Spectrum}

The infrared spectrum (Figure 3), was run on a Bruker IFS 66/S Fourier Transform Infrared Spectrometer (FT-IR), utilizing the ATR (Attenuated Total Reflectance) technique. The sample (powder or crystal) was directly placed on a germanium crystal and the spectrum was obtained using a detector (MCT). The absorption bands are expressed in wavenumber $\left(\mathrm{cm}^{-1}\right)$ with a resolution of $1 \mathrm{~cm}^{-1}$.

\section{4. ${ }^{1} \mathrm{H}$ and ${ }^{13} \mathrm{C}$ Spectra}

${ }^{1} \mathrm{H}$ and ${ }^{13} \mathrm{C}-\mathrm{NMR}$ spectra (figures 4 and 5) were recorded on a Bruker AMX-400 spectrometer at 400 and $100 \mathrm{MHz}$ respectively, using TMS as internal standard (chemical shifts in $\delta \mathrm{ppm}, \mathrm{J}$ in $\mathrm{Hz}$ ) and DMSO-d6 as solvent.

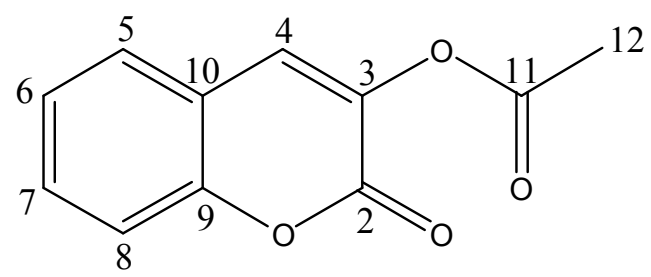

Figure 1. Atomic numbering scheme for analyzing spectra.

\subsection{Crystal structure Analysis}

Diffraction data were gathering using a Rigaku Oxford Diffraction SuperNova, Dual, $\mathrm{Cu}$ at zero, AtlasS2 diffractometer [10] utilizing a mirror monochromator and $\mathrm{Cu}$ $K \alpha$ radiation $(\lambda=1.54184 \AA)$ at $298 \mathrm{~K}$. The raw structure was determined by direct methods with SIR2014 [11] incorporated in the WinGX [12] package. The refinement was performed by full-matrix least squares method on the positional and anisotropic temperature parameters of the nonhydrogen atoms utilizing 137 crystallographic parameters with SHELXL2014 program [13]. All H atoms were geometrically optimized $[\mathrm{C}-\mathrm{H}=0.93$ (aromatic), $0.96 \AA$ (methyl)] and refined as riding model approximation with Uiso (H) constrained to 1.2 (aromatic group) or 1.5 (methyl group) times Ueq of the respective parent atom. Data gathering, cell refinement and data reduction are by CrysAlis PRO [10]. The general-purpose crystallographic tool PLATON [14] was used for the structure analysis and presentation of the results. Information on the data gathering conditions and the parameters of the refinement process are summarized in Table 1.

\subsection{Hirshfeld Surface}

The Hirshfeld surface analysis is a reliable tool to examine intermolecular associations of the molecules in a crystal packing. This tool implemented in Crystal Explorer 3.1 software [15] is used in this work to compute Hirshfeld surfaces map and 2-D fingerprint plots of 2-oxo-2Hchromen-3-yl acetate with a standard (high) surface resolution.

\subsection{Computational Procedures}

The geometry optimization of compound (I) was computed employing the density functional theory (DFT) and the frequency calculations both using restricted RB3LYP exchange correlation functional, the restricted Hartree-Fock (RHF) and the exchange component of Perdew and Wang's 1991 functional B3PW91 methods. The four methods were performed with the GAUSSIAN09 program package [16] utilizing the structure originating from crystallographic study as the starting structure for the computations. Further, $6-311^{++} \mathrm{G}(\mathrm{d}, \mathrm{p})$ basis set is applied to all calculation methods. 


\section{Results and Discussion}

\subsection{Spectra Analysis}

\subsubsection{Electrospray Ionisation Mass Spectrum}

In the mass spectrum (figure 2), the peaks detected at $\mathrm{m} / \mathrm{z}$ 205, 222 and 227 due to the pseudo-molecular ion $[\mathrm{M}+\mathrm{H}]^{+}$, the ammonium adduct $\left[\mathrm{M}+\mathrm{NH}_{4}\right]^{+}$and the sodium adduct $[\mathrm{M}+\mathrm{Na}]^{+}$successively, confirmed the molecular weight of 204 g. $\mathrm{mol}^{-1}$ which is in accordance with the chemical formula $\mathrm{C}_{11} \mathrm{H}_{8} \mathrm{O}_{4}$. The black dot observe in the spectrum indicates an ion that is not specific to the sample.

\subsubsection{Infrared (FT-IR) Spectrum}

The FT-IR spectrum of compound I (figure 3) highlighted the main characteristic absorption bands: $1776.7 \mathrm{~cm}^{-1}(\mathrm{C}=\mathrm{O}$, ester); $1731.8 \mathrm{~cm}^{-1}$ ( $\mathrm{C}=\mathrm{O}$, lactone); $1189.8 \mathrm{~cm}^{-1}$ (C-O, ester); $1081.7 \mathrm{~cm}^{-1}$ (C-O, lactone); $3064 \mathrm{~cm}^{-1}$ (C-H, aromatic) and $1607.4 \mathrm{~cm}^{-1}(\mathrm{C}=\mathrm{C}$, aromatic $)$.

\subsection{3. ${ }^{1} \mathrm{H}-\mathrm{NMR}$ Spectrum}

The analysis (chemical shifts and coupling constants) of the ${ }^{1} \mathrm{H}$ NMR spectrum (figures 4) showed six signals with five in the range 6-8.5 ppm due to aromatic hydrogens, the three equivalent methyl protons appeared unambiguously at $2.49 \mathrm{ppm}$.

${ }^{1} \mathrm{H}-\mathrm{NMR}$ (DMSO-d6, $400 \mathrm{MHz}, \delta \mathrm{ppm}$ ): 8.00 (s, 1H, H-4); $7.72(\mathrm{dd}, 1 \mathrm{H}, J=8.0,2.1 \mathrm{~Hz}, \mathrm{H}-5) ; 7.39$ (td, 1H, $J=8.0,2.0$ $\mathrm{Hz}, \mathrm{H}-6) ; 7.62$ (td, 1H, J=8.0, $2.1 \mathrm{~Hz}, \mathrm{H}-7) ; 7.46$ (d, 1H, $J=8.0 \mathrm{~Hz}, \mathrm{H}-8) ; 2.49$ (s, 3H, H-12).

\subsection{4. ${ }^{13}$ C-NMR Spectrum}

As expected, eleven peaks were observed in the ${ }^{13} \mathrm{C} N \mathrm{NMR}$ spectrum (figure 5).

${ }^{13} \mathrm{C}-\mathrm{NMR}$ (DMSO-d6, $\left.100 \mathrm{MHz}, \delta \mathrm{ppm}\right): 156.08$ (C-2), 135.33 (C-3), 125.04 (C-4), 131.47 (C-5), 128.44 (C-6), 131.68 (C-7), 116.16 (C-8), 151.52 (C-9), 118.27 (C-10), 168.19 (C-11), 20.12 (C-12).

\subsubsection{DEPT-135 Spectrum}

The spectrum of compound (I) (Figure 6) showed six positive peaks suggesting the five aromatic tertiary carbons and the shielded primary carbon of methyl group. It is also interesting to note that quaternary carbons (C-2, C-3, C-9, C10 and $\mathrm{C}-11)$ disappeared.

${ }^{13} \mathrm{C}$ (DEPT-135)-NMR (DMSO-d6, $\left.100 \mathrm{MHz}, \delta \mathrm{ppm}\right)$ : 125.04 (C-4), 131.47 (C-5), 128.44 (C-6), 131.68 (C-7), 116.16 (C-8), 20.12 (C-12).

\subsubsection{Conclusion of Spectra Analysis}

The overlaying of the spectrometric outcomes originating from the spectral analysis validates the depicted coumarin molecule in Figure 1.

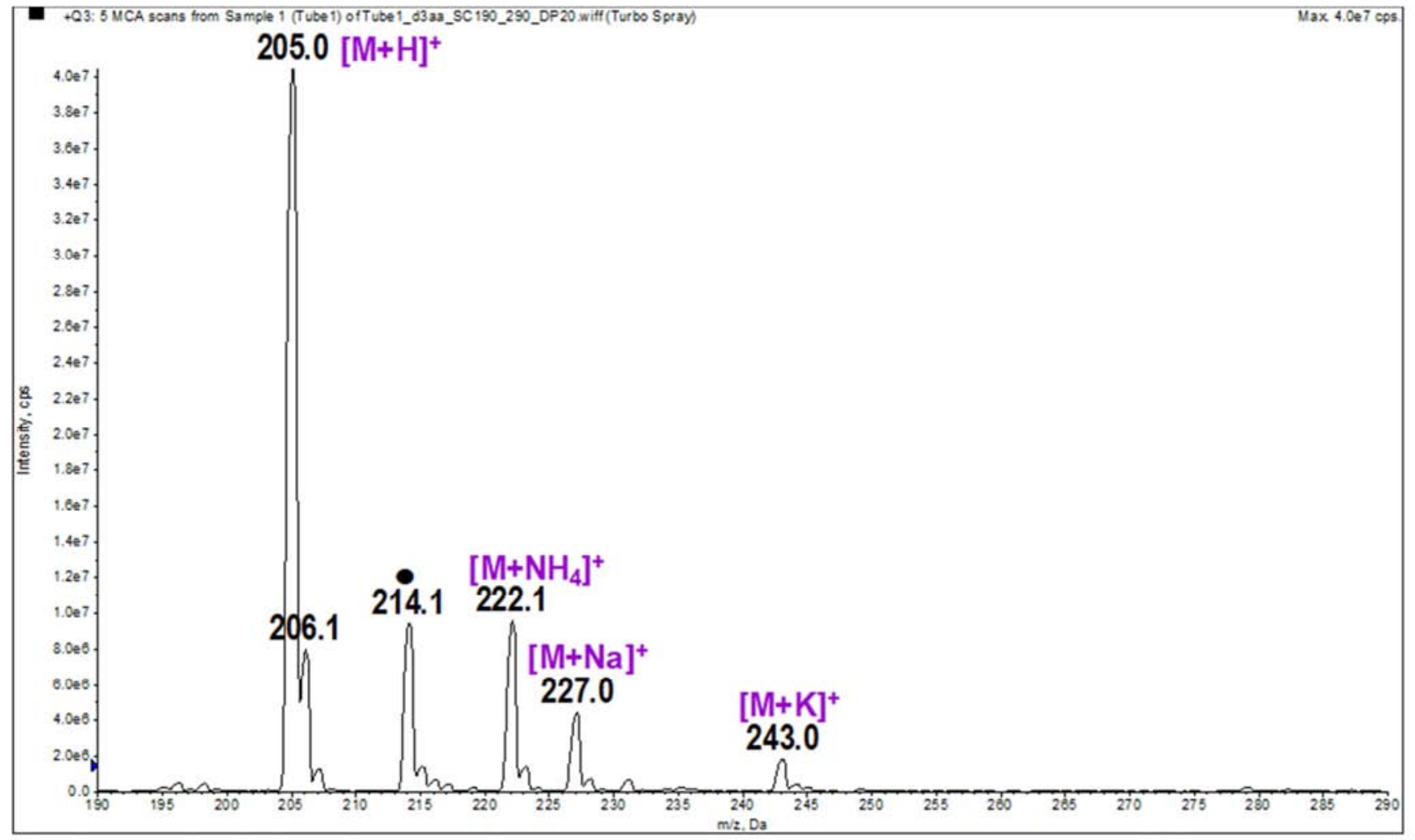

Figure 2. Experimental ESI-MS spectrum. 


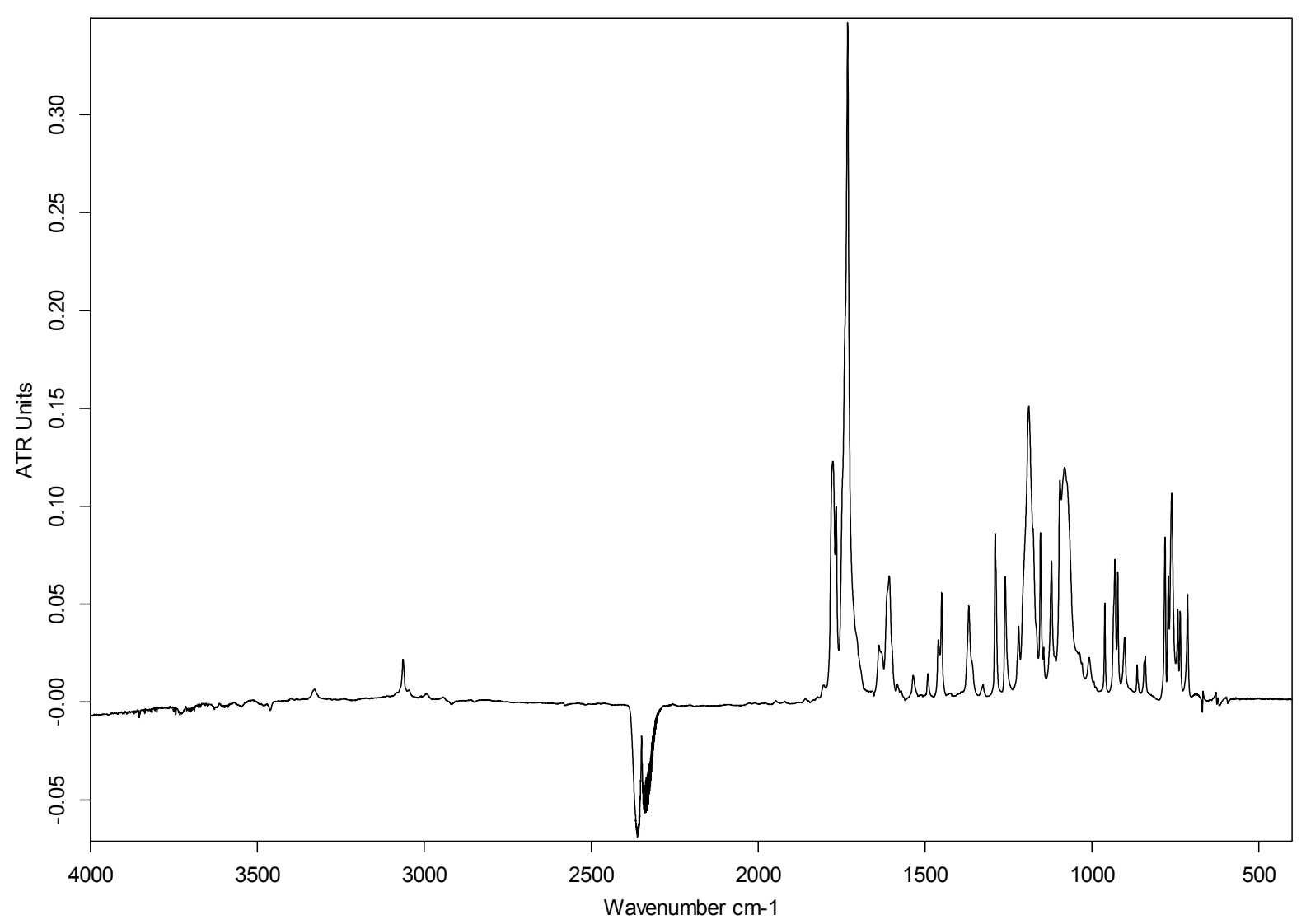

Figure 3. Experimental FT-IR spectrum.

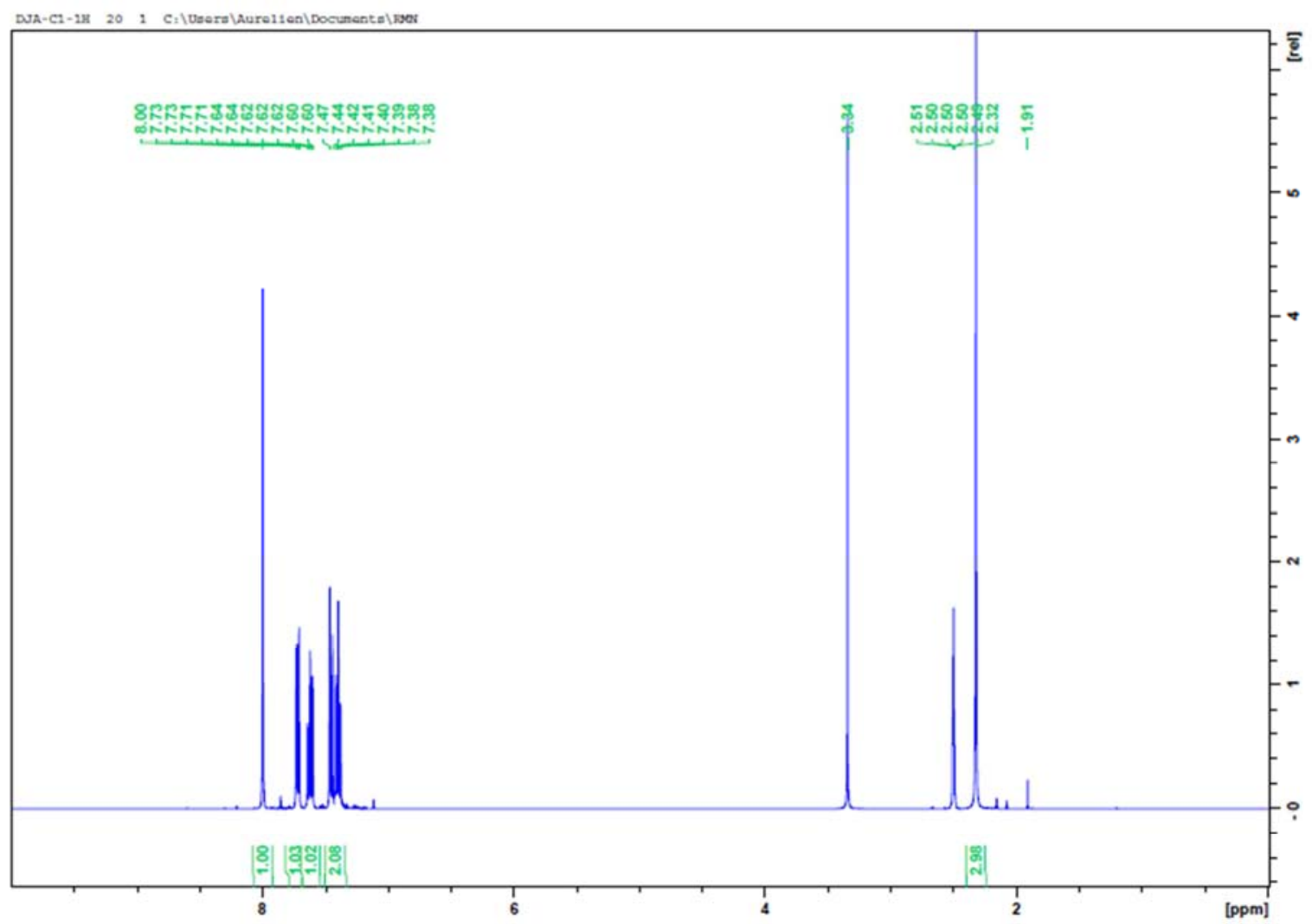

Figure 4. Experimental ${ }^{1} H$-NMR Spectrum. 


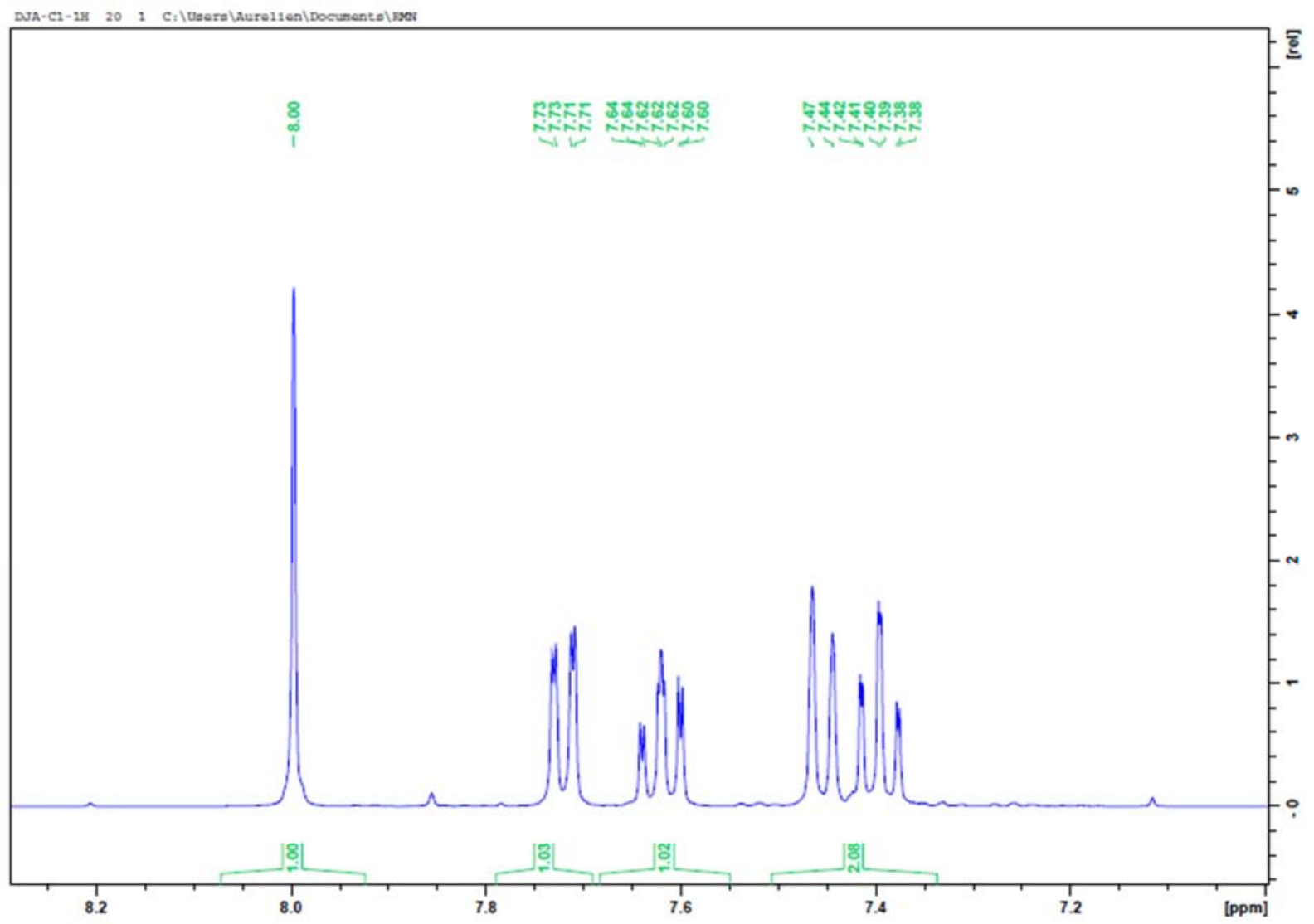

Figure 5. Experimental ${ }^{1} H-N M R$ Spectrum: enlargement of aromatic ring.

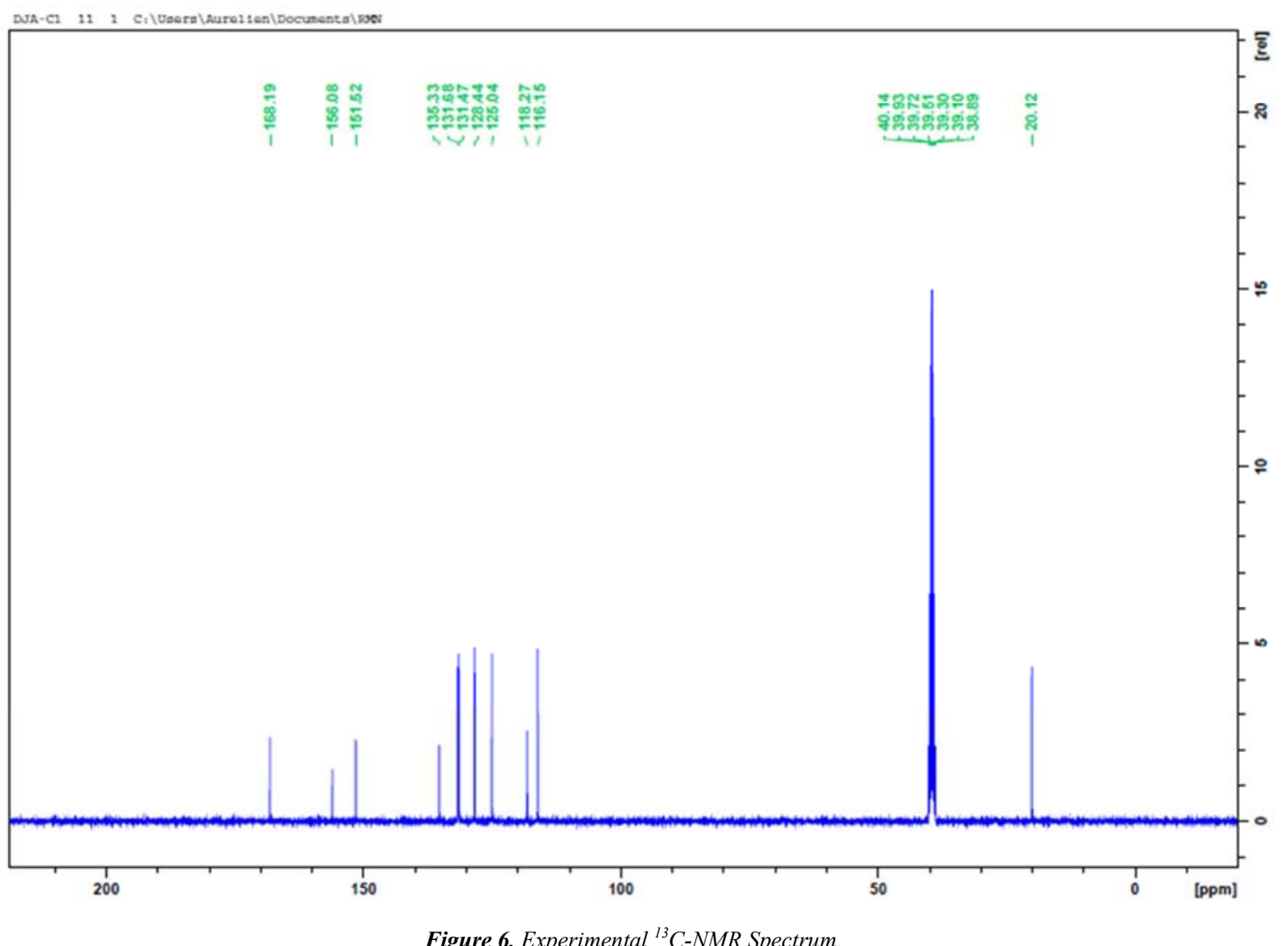

Figure 6. Experimental ${ }^{13} C-N M R$ Spectrum. 


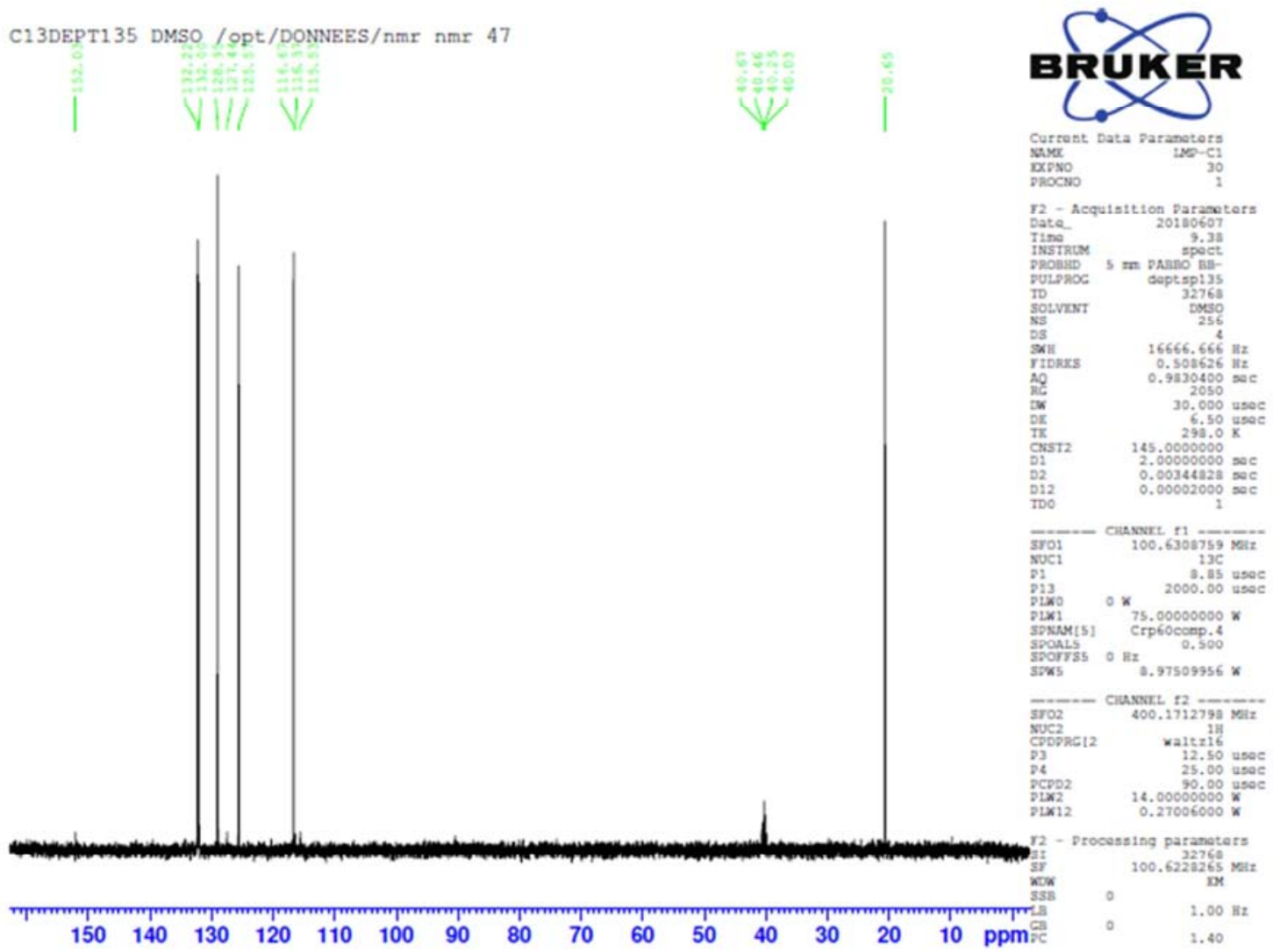

Figure 7. Experimental DEPT-135 Spectrum.

\subsection{Structural Description}

The molecular structure of (I) is represented in Figure 7.

\subsubsection{Geometry and Conformation}

The positional parameters, bond distances, bond angles and torsion angles are summarized in Tables 3, 4, 5 and 6. The analysis of the geometrical parameters are based on the comparison of these parameters with those from standard values [17]. The results of the investigation reveal their good compliance with the expected standard values despite the slight asymmetry found in the electronic distribution of the pyrone ring generating C2-C3 [1.334 (2) $\AA]$ and C1-C2 [1.461 (2) $\AA$ ] bond distances less and greater respectively than those expected for Car-Car bond. This demonstrates that the electron density is primarily situated in the $\mathrm{C} 2-\mathrm{C} 3$ bond of the pyrone ring, as observed in other coumarin derivatives $[18,19]$.

Table 1. Crystal data and details of the structure determination.

\begin{tabular}{|c|c|c|c|}
\hline chemical formula & $\mathrm{C}_{11} \mathrm{H}_{8} \mathrm{O}_{4}$ & Theta range for data collection $\left[{ }^{\circ}\right]$ & $4.979-70.901$ \\
\hline Formula weight & 204.17 & Crystal size $\left[\mathrm{mm}^{3}\right]$ & $0.33 \times 0.14 \times 0.06$ \\
\hline Temperature $[\mathrm{K}]$ & 298 & Index ranges & $-17 \leq \mathrm{h} \leq 17 ;-8 \leq \mathrm{k} \leq 8 ;-21 \leq 1 \leq 20$ \\
\hline Wavelength $\lambda[\AA]$ & 1.54184 & Refections collected & 18587 \\
\hline Crystal system & Orthorhombic & Absorption coefficient $\left[\mathrm{mm}^{-1}\right]$ & 0.96 \\
\hline Space group & Pbca & Theta full $\left[{ }^{\circ}\right]$ & 67.684 \\
\hline Unit cell dimensions & & $\mathrm{F}(000)$ & 848 \\
\hline $\mathrm{a}[\AA]$ & $14.6770(1)$ & Refinement method & Full-matrix least squares on $\mathrm{F}^{2}$ \\
\hline $\mathrm{c}[\AA]$ & $17.6767(2)$ & Goodness of fit & 1.07 \\
\hline$\alpha\left[^{\circ}\right]$ & 90 & Final $\mathrm{R}$ indices $\left[\mathrm{F}^{2}>2.0 \sigma\left(\mathrm{F}^{2}\right)\right]$ & $\mathrm{R}_{1}=0.038, \mathrm{wR}_{1}=0.116$ \\
\hline$\beta\left[^{\circ}\right]$ & 90 & Density calculated $\left[\mathrm{g} . \mathrm{cm}^{-3}\right]$ & 1.471 \\
\hline$\gamma\left[{ }^{\circ}\right]$ & 90 & Independent reflections & 1768 \\
\hline Volume $\left[\AA^{3}\right]$ & $1844.08(4)$ & $\mathrm{R}_{\text {int }}$ & 0.028 \\
\hline $\mathrm{Z}$ & 8 & $\mathrm{R}$ indices (all data) & 0.0395 \\
\hline $\begin{array}{l}\text { Crystal description- } \\
\text { crystal colour }\end{array}$ & Prism Yellow & $\Delta \rho_{\max }, \Delta \rho_{\min }\left(\mathrm{e} \AA^{-3}\right)$ & $0.23,-0.17$ \\
\hline Extinction correction: & None & $(\Delta / \sigma)_{\max }$ & $<0.001$ \\
\hline Diffractometer & $\begin{array}{l}\text { SuperNova, Dual, } \mathrm{Cu} \\
\text { at zero, AtlasS2 }\end{array}$ & Absorption correction & $\begin{array}{l}\text { multi-scan; CrysAlisPro 1.171.38.43 (Rigaku Oxford Diffraction, } \\
\text { 2015) Empirical absorption correction using spherical harmonics, } \\
\text { implemented in SCALE3 ABSPACK scaling algorithm. }\end{array}$ \\
\hline
\end{tabular}




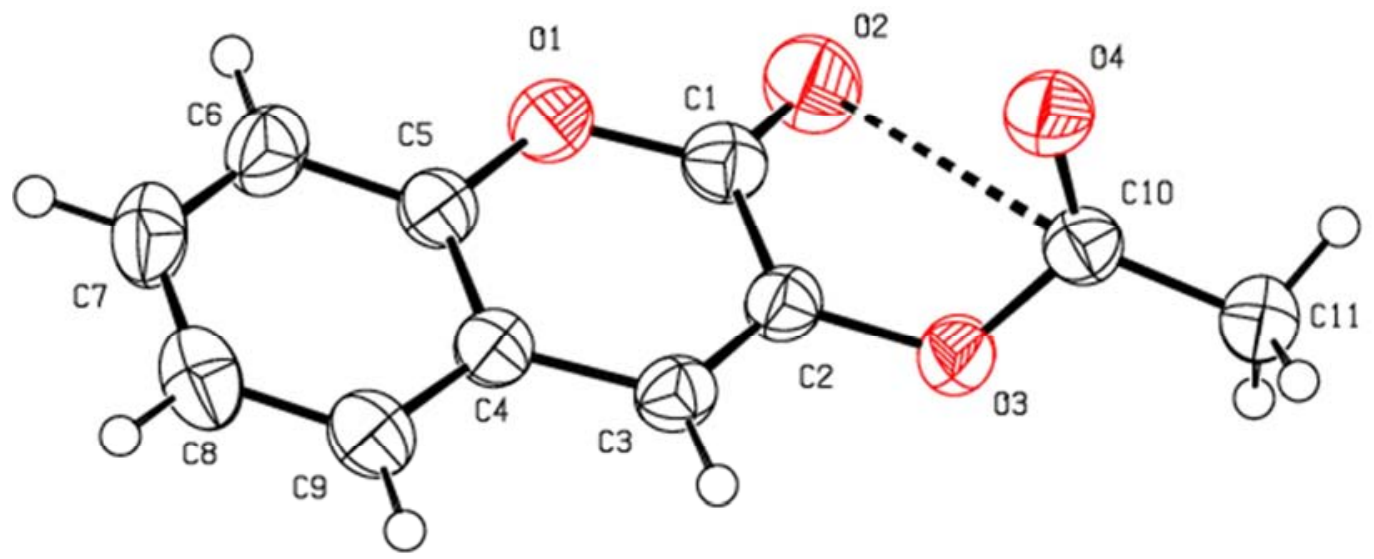

Figure 8. An ORTEP [12] view of (I) with Atomic numbering scheme. Dashed lines denotes short intramolecular contact.

In compound (I), the coumarin moiety is planar (r.m.s deviation $=0.037 \AA$ ) and makes an angle of $22.05(6)^{\circ}$ with respect to the pseudo five membered $\mathrm{O} 2 \mathrm{C} 1 \mathrm{C} 2 \mathrm{O} 3 \mathrm{C} 10$ ring established by the short intramolecular $\mathrm{O} 2 \ldots \mathrm{C} 10=3.1385$ (15) $\AA$ contact (figure 8 ). The conformation of this pseudo fivemembered ring is an envelope as judged by the Puckering parameters $\left(\mathrm{Q}=0.6405\right.$ (12) $\AA$ and $\Phi=319.00$ (11) $\left.{ }^{\circ}\right)$ [20]. The resulting tricycle compound is not coplanar as demonstrated by the angle between the coumrin moiety and the pseudo five-membered ring $\left(22.05(6)^{\circ}\right)$.

\subsubsection{Supramolecular Features}

In the crystal, $\mathrm{C} 11-\mathrm{H} 11 \mathrm{C} \ldots \mathrm{O} 3$ hydrogen bonds (table 2) connect adjacent molecules in an infinite chain along [010] direction (Figure 9). In addition, Platon [14] analysis reveals short contacts [H11A...O2 $(1 / 2-\mathrm{x},-1 / 2+\mathrm{y}, \mathrm{z})=2.65 \AA]$ and $[\mathrm{C} 2 \ldots 2(-\mathrm{x}, 2-\mathrm{y},-\mathrm{z})=3.2313(16) \AA]$ with distances less than the sum of the van der Waals radii which insure the stability of compound (I).

Table 2. Hydrogen-bond geometry $\left(\AA{ }^{\circ}{ }^{\circ}\right)$.

\begin{tabular}{|c|c|c|c|c|}
\hline D-H...A & $\mathbf{D}-\mathbf{H}$ & H...A & D...A & D-H...A \\
\hline $\mathrm{C} 11-\mathrm{H} 11 \mathrm{C} \ldots \mathrm{O}^{\mathrm{i}}$ & 0.96 & 2.59 & $3.441(2)$ & 147.9 \\
\hline Symmetry code: (i) $-x+1 / 2, y+1 / 2, z$ & & & & \\
\hline
\end{tabular}

Table 3. Fractional atomic coordinates and isotropic or equivalent isotropic displacement parameters $\left(A^{2}\right)$.

\begin{tabular}{|c|c|c|c|c|}
\hline Atom & $\mathbf{x}$ & $\mathbf{y}$ & $\mathbf{z}$ & $U_{\text {iso }} * / U_{\text {eq }}$ \\
\hline $\mathrm{O} 3$ & $0.36063(6)$ & $0.49673(12)$ & $0.00918(5)$ & $0.0421(3)$ \\
\hline $\mathrm{O} 1$ & $0.53198(6)$ & $0.67749(11)$ & $0.13964(5)$ & 0.0438 \\
\hline $\mathrm{C} 4$ & $0.52874(7)$ & $0.33942(16)$ & $0.15009(7)$ & $0.0369(3)$ \\
\hline C6 & $0.63015(9)$ & $0.5293(2)$ & $0.22686(8)$ & $0.0492(3)$ \\
\hline H6 & 0.6521 & 0.6469 & 0.2411 & $0.059 *$ \\
\hline $\mathrm{O} 2$ & $0.44319(7)$ & $0.82796(13)$ & $0.05919(6)$ & 0.0568 \\
\hline $\mathrm{C} 11$ & $0.21573(9)$ & 0.55735 (19) & $-0.04229(8)$ & 0.0485 \\
\hline $\mathrm{C} 7$ & $0.66341(9)$ & $0.3678(2)$ & $0.26024(8)$ & $0.0535(4)$ \\
\hline $\mathrm{C} 9$ & $0.56541(8)$ & 0.17779 (18) & $0.18333(8)$ & 0.0454 \\
\hline H9 & 0.5447 & 0.0597 & 0.1685 & $0.054 *$ \\
\hline $\mathrm{C} 3$ & $0.45608(8)$ & $0.33576(16)$ & $0.09554(7)$ & 0.0379 \\
\hline $\mathrm{H} 3$ & 0.4309 & 0.2217 & 0.0803 & $0.046^{*}$ \\
\hline $\mathrm{C} 10$ & $0.27554(8)$ & $0.56698(16)$ & $0.02550(7)$ & $0.0390(3)$ \\
\hline $\mathrm{C} 2$ & $0.42497(8)$ & $0.49711(16)$ & $0.06687(6)$ & 0.0368 \\
\hline $\mathrm{O} 4$ & $0.25572(6)$ & $0.62334(14)$ & $0.08671(5)$ & 0.0538 \\
\hline $\mathrm{C} 8$ & $0.63196(9)$ & $0.1926(2)$ & $0.23786(8)$ & $0.0521(4)$ \\
\hline $\mathrm{H} 8$ & 0.6560 & 0.0844 & 0.2598 & $0.062 *$ \\
\hline $\mathrm{H} 7$ & 0.7073 & 0.3767 & 0.2981 & $0.064 *$ \\
\hline H11A & 0.1792 & 0.4454 & -0.0400 & $0.073 *$ \\
\hline H11B & 0.2526 & 0.5546 & -0.0871 & $0.073^{*}$ \\
\hline $\mathrm{H} 11 \mathrm{C}$ & 0.1767 & 0.6657 & -0.0435 & $0.073^{*}$ \\
\hline
\end{tabular}


Table 4. Experimental and calculated bond lengths with $6-311^{++} G(d, p)$ basis set $(\AA)$.

\begin{tabular}{lllll}
\hline Bond & X-Ray & DFT/RB3LYP/ & FREQ/RB3LYP & HF \\
\hline O3-C10 & $1.3756(14)$ & 1.3879 & 1.3757 & 1.3522 \\
O3-C2 & $1.3899(14)$ & 1.3783 & 1.3898 & 1.3612 \\
O1-C1 & $1.3719(15)$ & 1.3836 & 1.3724 & 1.3411 \\
O1-C5 & $1.3787(15)$ & 1.3677 & 1.3789 & 1.3550 \\
C3-C2 & $1.3343(16)$ & 1.3455 & 1.3339 & 1.3223 \\
C3-C4 & $1.4380(16)$ & 1.4392 & 1.4375 & 1.4524 \\
O2-C1 & $1.1970(15)$ & 1.2005 & 1.1965 & 1.1769 \\
C4-C5 & $1.3899(16)$ & 1.4043 & 1.3903 & 1.3761 \\
C4-C9 & $1.3982(17)$ & 1.4060 & 1.3987 & 1.3612 \\
O4-C10 & $1.1899(15)$ & 1.1964 & 1.1900 & 1.3949 \\
C2-C1 & $1.4606(16)$ & 1.4694 & 1.4612 & 1.1991 \\
C5-C6 & $1.3852(18)$ & 1.4060 & 1.3850 & 1.4020 \\
C9-C8 & $1.3762(19)$ & 1.3855 & 1.3758 & 1.4034 \\
C6-C7 & $1.380(2)$ & 1.3855 & 1.3799 & 1.3765 \\
C10-C11 & $1.4870(17)$ & 1.4997 & 1.4877 & 1.3839 \\
C8-C7 & $1.386(2)$ & 1.4011 & 1.3855 & 1.3773 \\
\hline
\end{tabular}

Table 5. Experimental and computed bond angles with $6-311^{++} G(d, p)$ basis set $\left(^{\circ}\right)$.

\begin{tabular}{|c|c|c|c|c|c|}
\hline Bond angle & X-Ray & DFT/RB3LYP & FREQ/ RB3LYP & RHF & B3PW91 \\
\hline $\mathrm{C} 10-\mathrm{O} 3-\mathrm{C} 2$ & $117.53(9)$ & 117.14 & 117.52 & 118.09 & 116.61 \\
\hline $\mathrm{C} 1-\mathrm{O} 1-\mathrm{C} 5$ & $122.46(9)$ & 123.37 & 122.47 & 124.10 & 123.38 \\
\hline $\mathrm{C} 2-\mathrm{C} 3-\mathrm{C} 4$ & $119.52(10)$ & 120.16 & 119.57 & 119.76 & 120.01 \\
\hline $\mathrm{C} 5-\mathrm{C} 4-\mathrm{C} 9$ & $118.23(11)$ & 118.41 & 118.18 & 118.71 & 118.46 \\
\hline $\mathrm{C} 5-\mathrm{C} 4-\mathrm{C} 3$ & $118.23(10)$ & 117.71 & 118.25 & 117.54 & 117.68 \\
\hline $\mathrm{C} 9-\mathrm{C} 4-\mathrm{C} 3$ & $123.54(11)$ & 123.87 & 123.56 & 123.75 & 123.86 \\
\hline $\mathrm{C} 3-\mathrm{C} 2-\mathrm{O} 3$ & $120.62(10)$ & 121.72 & 120.69 & 122.76 & 121.82 \\
\hline $\mathrm{C} 3-\mathrm{C} 2-\mathrm{C} 1$ & $122.71(11)$ & 122.35 & 122.68 & 121.81 & 122.29 \\
\hline $\mathrm{O} 3-\mathrm{C} 2-\mathrm{C} 1$ & $116.18(10)$ & 115.78 & 116.13 & 115.34 & 115.73 \\
\hline $\mathrm{O} 1-\mathrm{C} 5-\mathrm{C} 6$ & $117.15(11)$ & 117.40 & 117.19 & 117.41 & 117.34 \\
\hline $\mathrm{O} 1-\mathrm{C} 5-\mathrm{C} 4$ & 121.07 (11) & 121.11 & 121.04 & 121.02 & 121.24 \\
\hline $\mathrm{C} 6-\mathrm{C} 5-\mathrm{C} 4$ & $121.78(11)$ & 121.49 & 121.77 & 121.57 & 121.42 \\
\hline $\mathrm{O} 2-\mathrm{C} 1-\mathrm{O} 1$ & $118.14(11)$ & 119.11 & 118.12 & 120.13 & 119.17 \\
\hline $\mathrm{O} 2-\mathrm{C} 1-\mathrm{C} 2$ & $125.97(12)$ & 125.61 & 126.01 & 124.10 & 125.45 \\
\hline $\mathrm{O} 1-\mathrm{C} 1-\mathrm{C} 2$ & $115.88(10)$ & 115.26 & 115.86 & 115.76 & 115.37 \\
\hline $\mathrm{C} 8-\mathrm{C} 9-\mathrm{C} 4$ & $120.32(12)$ & 120.52 & 120.35 & 120.47 & 120.53 \\
\hline $\mathrm{C} 7-\mathrm{C} 6-\mathrm{C} 5$ & $118.79(12)$ & 118.97 & 118.81 & 118.83 & 118.99 \\
\hline $\mathrm{O} 4-\mathrm{C} 10-\mathrm{O} 3$ & $122.34(11)$ & 122.48 & 122.36 & 122.57 & 122.44 \\
\hline $\mathrm{O} 4-\mathrm{C} 10-\mathrm{C} 11$ & $127.15(11)$ & 127.42 & 127.15 & 126.74 & 127.40 \\
\hline $\mathrm{O} 3-\mathrm{C} 10-\mathrm{C} 11$ & $110.50(10)$ & 110.10 & 110.48 & 110.68 & 110.15 \\
\hline $\mathrm{C} 9-\mathrm{C} 8-\mathrm{C} 7$ & $120.32(12)$ & 120.68 & 120.32 & 119.71 & 119.91 \\
\hline
\end{tabular}

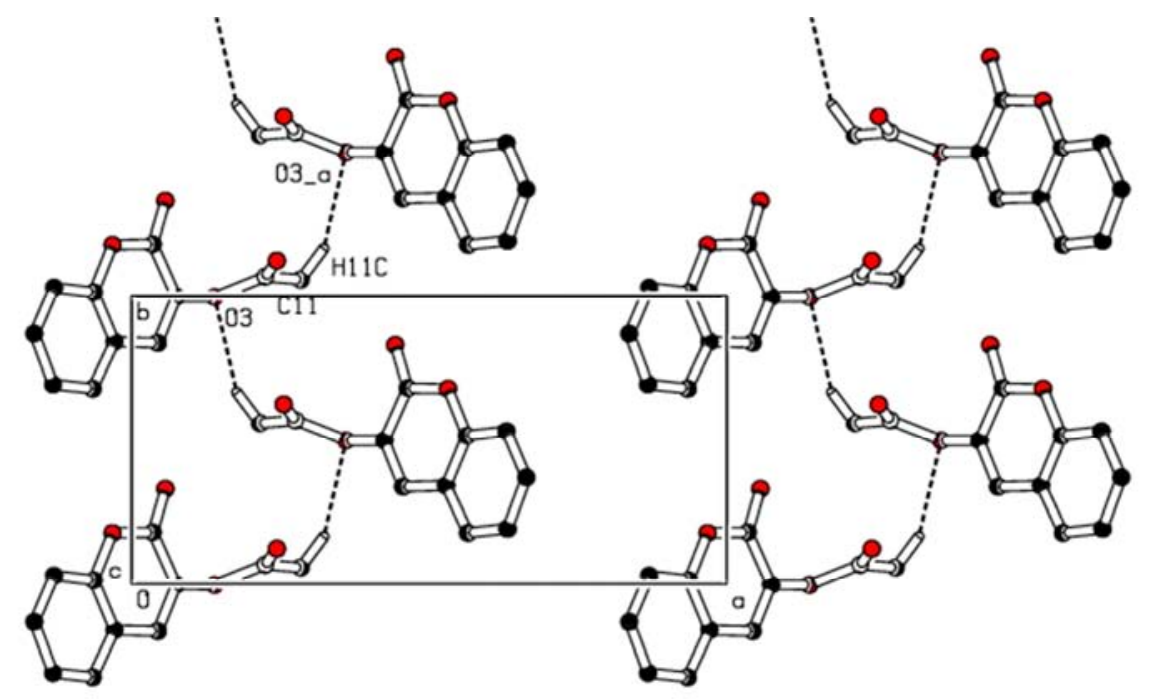

Figure 9. Part of the crystal packing showing hydrogen bonds (dashed lines) along [010]. H atoms not involved in hydrogen bonding have been omitted for clarity. 
Table 6. Experimental and computed torsion angles with $6-311^{++} G(d, p)$ basis set $\left(^{\circ}\right)$.

\begin{tabular}{|c|c|c|c|c|c|}
\hline Torsion angle & X-ray & DFT/RB3LYP & FREQ/ RB3LYP & RHF & B3PW91 \\
\hline $\mathrm{C} 2-\mathrm{C} 3-\mathrm{C} 4-\mathrm{C} 5$ & $0.76(17)$ & 0.54 & 0.76 & 0.35 & 0.59 \\
\hline $\mathrm{C} 2-\mathrm{C} 3-\mathrm{C} 4-\mathrm{C} 9$ & $179.76(11)$ & 179.82 & 179.77 & 179.76 & 179.76 \\
\hline $\mathrm{C} 4-\mathrm{C} 3-\mathrm{C} 2-\mathrm{O} 3$ & $174.17(10)$ & 176.27 & 174.15 & 177.03 & 176.33 \\
\hline $\mathrm{C} 4-\mathrm{C} 3-\mathrm{C} 2-\mathrm{C} 1$ & $2.54(18)$ & 0.87 & 2.55 & 0.74 & 1.06 \\
\hline $\mathrm{C} 10-\mathrm{O} 3-\mathrm{C} 2-\mathrm{C} 3$ & $113.77(12)$ & 112.31 & 113.74 & 108.57 & 112.94 \\
\hline $\mathrm{C} 10-\mathrm{O} 3-\mathrm{C} 2-\mathrm{C} 1$ & $-74.08(13)$ & -72.01 & -74.13 & -74.91 & -71.49 \\
\hline $\mathrm{C} 1-\mathrm{O} 1-\mathrm{C} 5-\mathrm{C} 6$ & $-177.98(10)$ & 179.64 & -177.99 & 179.85 & 179.58 \\
\hline $\mathrm{C} 1-\mathrm{O} 1-\mathrm{C} 5-\mathrm{C} 4$ & $1.39(17)$ & -0.07 & 1.43 & 0.08 & -0.13 \\
\hline $\mathrm{C} 9-\mathrm{C} 4-\mathrm{C} 5-\mathrm{O} 1$ & $178.21(10)$ & 179.72 & 178.18 & 179.77 & 179.70 \\
\hline $\mathrm{C} 3-\mathrm{C} 4-\mathrm{C} 5-\mathrm{O} 1$ & $-2.74(17)$ & -0.96 & -2.76 & -0.79 & -1.08 \\
\hline $\mathrm{C} 9-\mathrm{C} 4-\mathrm{C} 5-\mathrm{C} 6$ & $-2.45(18)$ & 0.01 & -2.43 & 0.00 & 0.00 \\
\hline $\mathrm{C} 3-\mathrm{C} 4-\mathrm{C} 5-\mathrm{C} 6$ & $176.61(11)$ & 179.34 & 176.63 & 179.45 & 179.22 \\
\hline $\mathrm{C} 5-\mathrm{O} 1-\mathrm{C} 1-\mathrm{O} 2$ & $-177.30(11)$ & -177.89 & -177.30 & -178.35 & -177.56 \\
\hline $\mathrm{C} 5-\mathrm{O} 1-\mathrm{C} 1-\mathrm{C} 2$ & $1.78(16)$ & 1.41 & 1.74 & 0.98 & 1.69 \\
\hline $\mathrm{C} 3-\mathrm{C} 2-\mathrm{C} 1-\mathrm{O} 2$ & $175.19(12)$ & 177.44 & 175.16 & 177.90 & 177.03 \\
\hline $\mathrm{O} 3-\mathrm{C} 2-\mathrm{C} 1-\mathrm{O} 2$ & $3.22(18)$ & 1.79 & 3.20 & 1.35 & 1.48 \\
\hline $\mathrm{C} 3-\mathrm{C} 2-\mathrm{C} 1-\mathrm{O} 1$ & $-3.81(17)$ & -1.82 & -3.79 & -1.40 & -2.16 \\
\hline $\mathrm{O} 3-\mathrm{C} 2-\mathrm{C} 1-\mathrm{O} 1$ & $-175.78(9)$ & -177.46 & -175.75 & -177.95 & -177.71 \\
\hline $\mathrm{C} 5-\mathrm{C} 4-\mathrm{C} 9-\mathrm{C} 8$ & $2.13(18)$ & -0.01 & 2.17 & 1.46 & -0.01 \\
\hline $\mathrm{C} 3-\mathrm{C} 4-\mathrm{C} 9-\mathrm{C} 8$ & $-176.87(11)$ & -179.29 & -176.84 & -179.43 & -179.18 \\
\hline $\mathrm{O} 1-\mathrm{C} 5-\mathrm{C} 6-\mathrm{C} 7$ & $-179.91(11)$ & -179.73 & -179.89 & -179.77 & -179.71 \\
\hline $\mathrm{C} 4-\mathrm{C} 5-\mathrm{C} 6-\mathrm{C} 7$ & $0.72(19)$ & -0.02 & 0.70 & 0.00 & -0.01 \\
\hline $\mathrm{C} 2-\mathrm{O} 3-\mathrm{C} 10-\mathrm{O} 4$ & $-1.43(17)$ & -12.62 & -1.35 & -11.93 & -12.64 \\
\hline $\mathrm{C} 2-\mathrm{O} 3-\mathrm{C} 10-\mathrm{C} 11$ & $179.59(10)$ & 167.64 & 179.61 & 168.55 & 167.65 \\
\hline $\mathrm{C} 4-\mathrm{C} 9-\mathrm{C} 8-\mathrm{C} 7$ & $-0.1(2)$ & 0.03 & -0.21 & 0.03 & 0.03 \\
\hline $\mathrm{C} 5-\mathrm{C} 6-\mathrm{C} 7-\mathrm{C} 8$ & $1.4(2)$ & 0.03 & 1.34 & 0.00 & 0.02 \\
\hline $\mathrm{C} 9-\mathrm{C} 8-\mathrm{C} 7-\mathrm{C} 6$ & $-1.7(2)$ & -0.03 & -1.59 & -0.02 & -0.03 \\
\hline
\end{tabular}

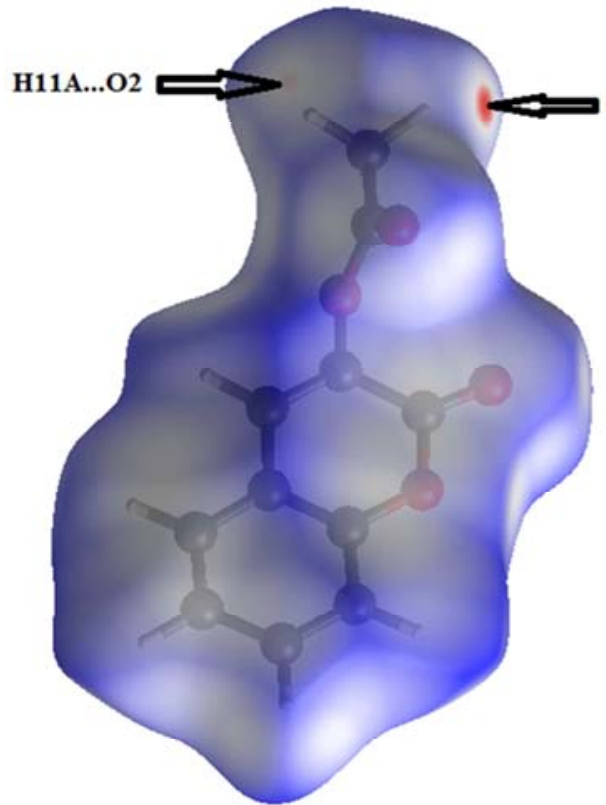

a)

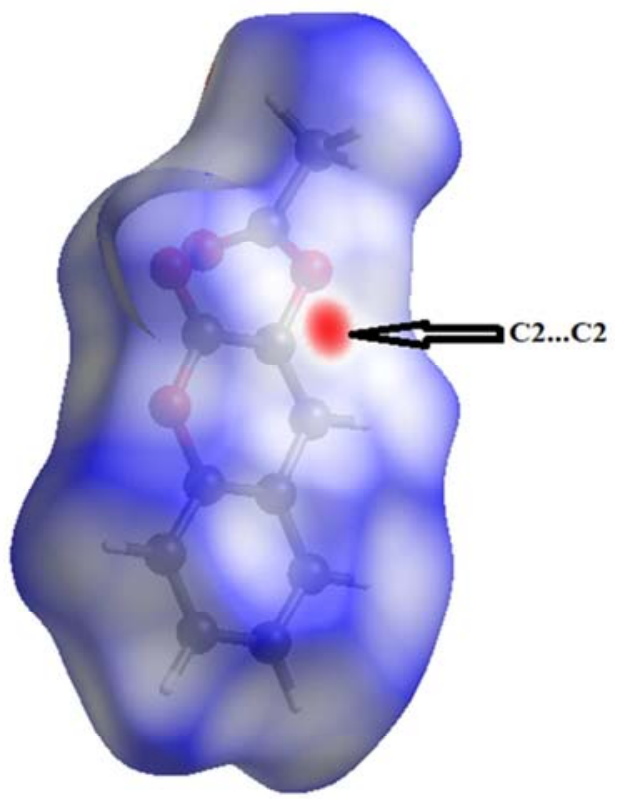

b)

Figure 10. Hirshfeld surfaces mapped over dnorm (-0.099 to 1.048 a.u.).

\subsection{Hirshfeld Surface Analysis}

The Hirshfeld surface analysis of compound (I) was computed employing a standard (high) surface resolution with the three-dimensional (3D) $d_{\text {norm }}$ surface schemed over a settled color scale of -0.099 (red) to 1.048 a.u. (blue). The resulting map shows two red tasks and one pale red task (negative $d_{\text {norm }}$ values), highlighting distances less than the sum of the van der Waals radii. These short contacts indicate intermolecular $\mathrm{C} 11-\mathrm{H} 11 \mathrm{C} \ldots \mathrm{O} 3 \quad(-\mathrm{x}+1 / 2, \quad \mathrm{y}+1 / 2, \quad \mathrm{z})$ hydrogen bond, C2...C2 (-x, 2-y, -z) and H11A...O2 (1/2-x,$1 / 2+y, \quad z)$ interactions between the surface and the surroundings areas. The mapping also points out white tasks revealing distances almost equal to the sum of the van der Waals radii, and blue areas with distances greater than the sum of the van der Waals radii. The surfaces are made transparent in order to show the molecule (Figures 10a and 
10b). Further, the colorful two-dimensional fingerprint (FP) plot is used to give information about the studied compound such as close contacts of atom pairs and the contributions from different contacts (figure 11). Hence, the blue tasks displayed near $\mathrm{de}=\mathrm{di} \simeq 1.8-2.0 \AA$ point out close $\mathrm{C}$... intermolecular contacts. The low contribution of these contacts $(2.6 \%)$ to the total Hirshfeld surface area suggests the absence of $\pi-\pi$ interactions [21] as forecasted by the crystallographic study. The most prominent type of contacts with $38.7 \%$ contribution to the overall surface contacts arises from $\mathrm{H} \ldots \mathrm{O} / \mathrm{O} \ldots \mathrm{H}$ interactions. These are displayed on the left side as blue spikes with the tip at de $+\mathrm{di}=2.6 \AA$ (Figure $11 \mathrm{~b}$ ), indicating $\mathrm{O} \ldots \mathrm{H}$ contacts. As generally found in organic chemistry, the H...H contacts have also a great contribution to Hirshfeld surface (28.7\%). These interactions are displayed in the central area of the $2 \mathrm{D}$ - fingerprint characterized by a blue tip spike at $\mathrm{de}+\mathrm{di}=2.4 \AA$ (Figure 11c) while the C...H/H...C plot $(20.2 \%)$ gives details on the intermolecular hydrogen bonds (Figure 11d). Other spots in the Hirshfeld surfaces indicating $\mathrm{C} \ldots \mathrm{O} / \mathrm{O} \ldots \mathrm{C}$ and $\mathrm{O} \ldots \mathrm{O}$ contacts are observed with contributions of 7.8 and $2.0 \%$, successively (Figures 11e and 11f).
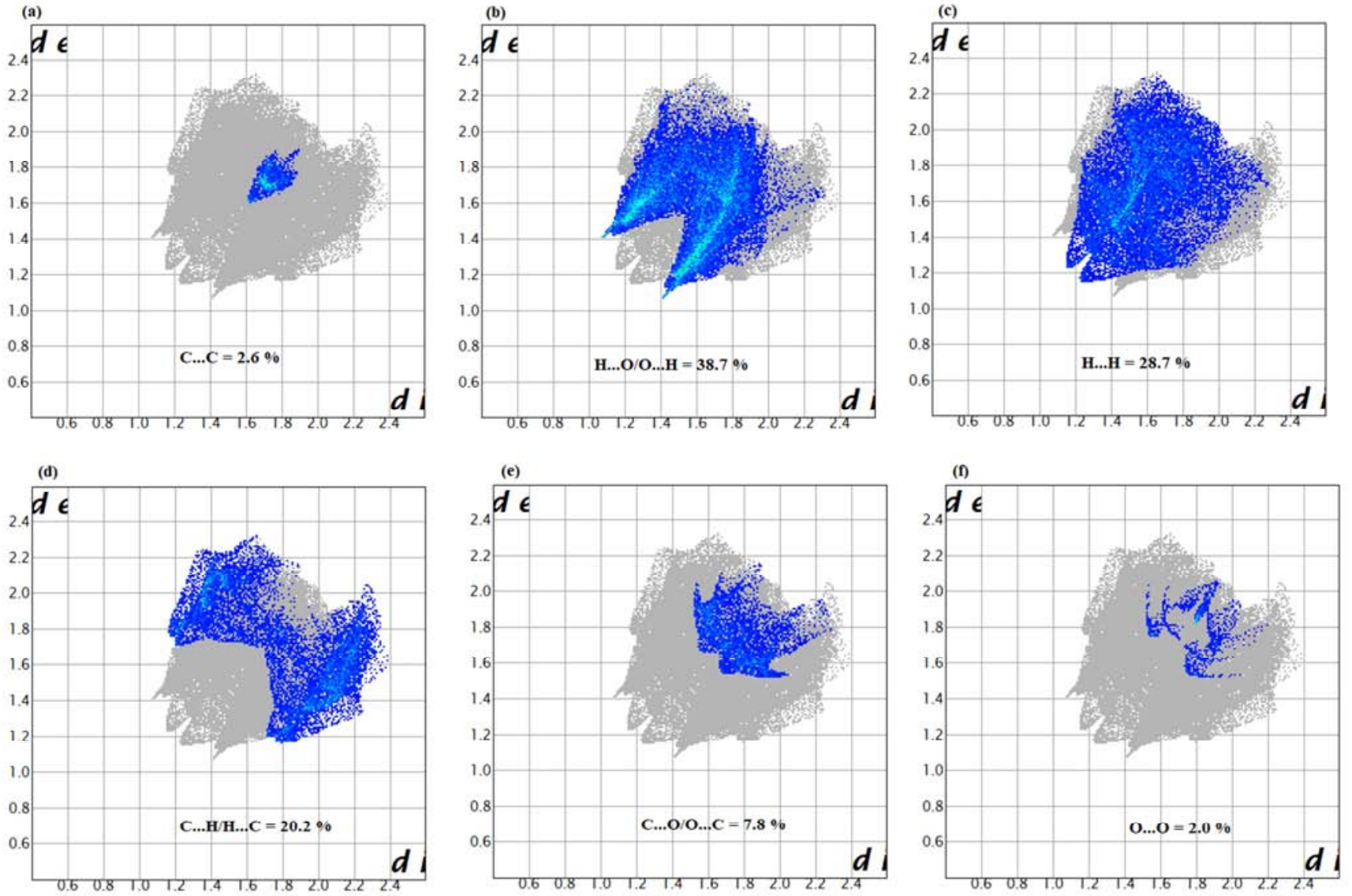

Figure 11. Atom-atom interactions and their relative contribution to the Hirshfeld surface.

Table 7. Comparison of the observed and calculated stretching vibrational spectra of compound (I).

\begin{tabular}{|c|c|c|}
\hline \multirow{2}{*}{ Vibrational mode } & \multirow{2}{*}{ Experimental IR, $\mathrm{cm}^{-1}$} & Calculated [B3LYP] 6-311 ${ }^{++}$G(d,p) \\
\hline & & scaled frequency, $\mathrm{cm}^{-1}$ \\
\hline $\bar{v}_{C-H(\text { aromatic })}$ & 3064 & 3162.82 \\
\hline $\bar{v}_{C=O(e s t e r)}$ & 1776.7 & 1824.96 \\
\hline $\bar{v}_{C=O(\text { lactone })}$ & 1731.8 & 1739.61 \\
\hline $\bar{v}_{C-O(e s t e r)}$ & 1189.8 & 1140.07 \\
\hline $\bar{v}_{C-O(\text { lactone })}$ & 1081.7 & 1091.01 \\
\hline $\bar{v}_{C=C(\text { aromatic })}$ & 1607.4 & 1646.73 \\
\hline
\end{tabular}

\subsection{Theoretical Calculations}

\subsubsection{Comparison of Geometrical Parameters and Vibrational Frequencies}

The molecular geometry evidenced by X-ray single crystal structure determination of (I) are compared to those obtained from Quantum Chemical Calculations. An inspection of the calculated bond lengths and bond angles reveals a strong correlation with the experiment values as judged by the rootmean-square deviation of $0.011 \AA$ (DFT/RB3LYP), $0.016 \AA$ (HF), $0.0004 \AA$ (FREQ/RB3LYP) and $0.009 \AA$ (B3PW91) for bond lengths and $0.5^{\circ}$ (DFT/RB3LYP), $0.9^{\circ}$ (RHF), $0.03^{\circ}$ (FREQ/RB3LYP) and $0.5^{\circ}$ (B3PW91) for bond angles 
(Tables 4 and 5). These demonstrate that the method giving the best results is FREQ / RB3LYP and the ranking of these methods in descending order is FREQ / RB3LYP, B3PW91, DFT / RB3LYP and HF for bond lengths and bond angles. In addition, the computed torsion angles' analysis highlights the flatness of the coumarin ring system which is in good accordance with the crystallographic forecast, despite the difference observed in the torsion $\mathrm{C} 2-\mathrm{O} 3-\mathrm{C} 10-\mathrm{O} 4$ angle between this crystallography method $\left(-1.43(17)^{\circ}\right)$ and the theoretical calculations ones (B3PW91, DFT / RB3LYP and $\mathrm{HF}$ ) which generate values close to $12^{\circ}$ (Table 6 ).

The theoretical spectrum (figure 13) was carried out using FREQ/RB3LYP/6-311G ${ }^{++}(\mathrm{d}, \mathrm{p})$ method for optimizing geometries. Some vibrational modes assigned by Avogadro software [22] and their corresponding experimental values are summarized in Table 7. Comparison of the two set of data is performed with the statistic methods using the linear regression method. The coefficient of determination of the model established by calculated values vs experimental ones is very close to unity $\left(\mathrm{R}^{2}=0.9926\right)$ indicating a better fit for the model (figure 12). Thus, the model is suitable for predicting experimental infrared frequencies with very good precision.

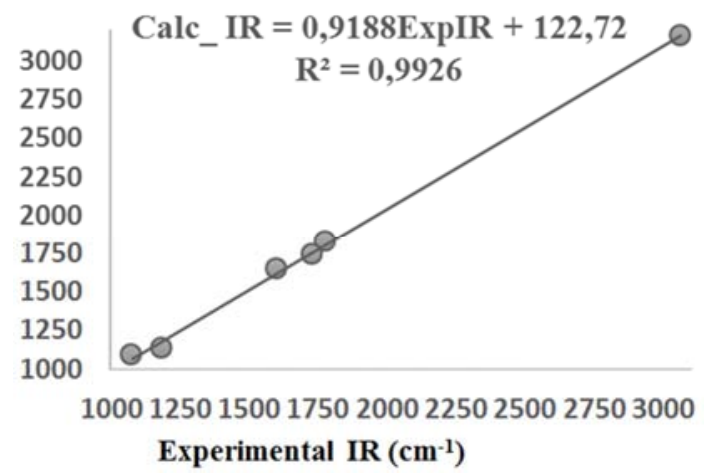

Figure 12. Correlation graphic between the experimental and theoretical vibration frequencies of compound (I) $\left(\mathrm{cm}^{-1}\right)$.

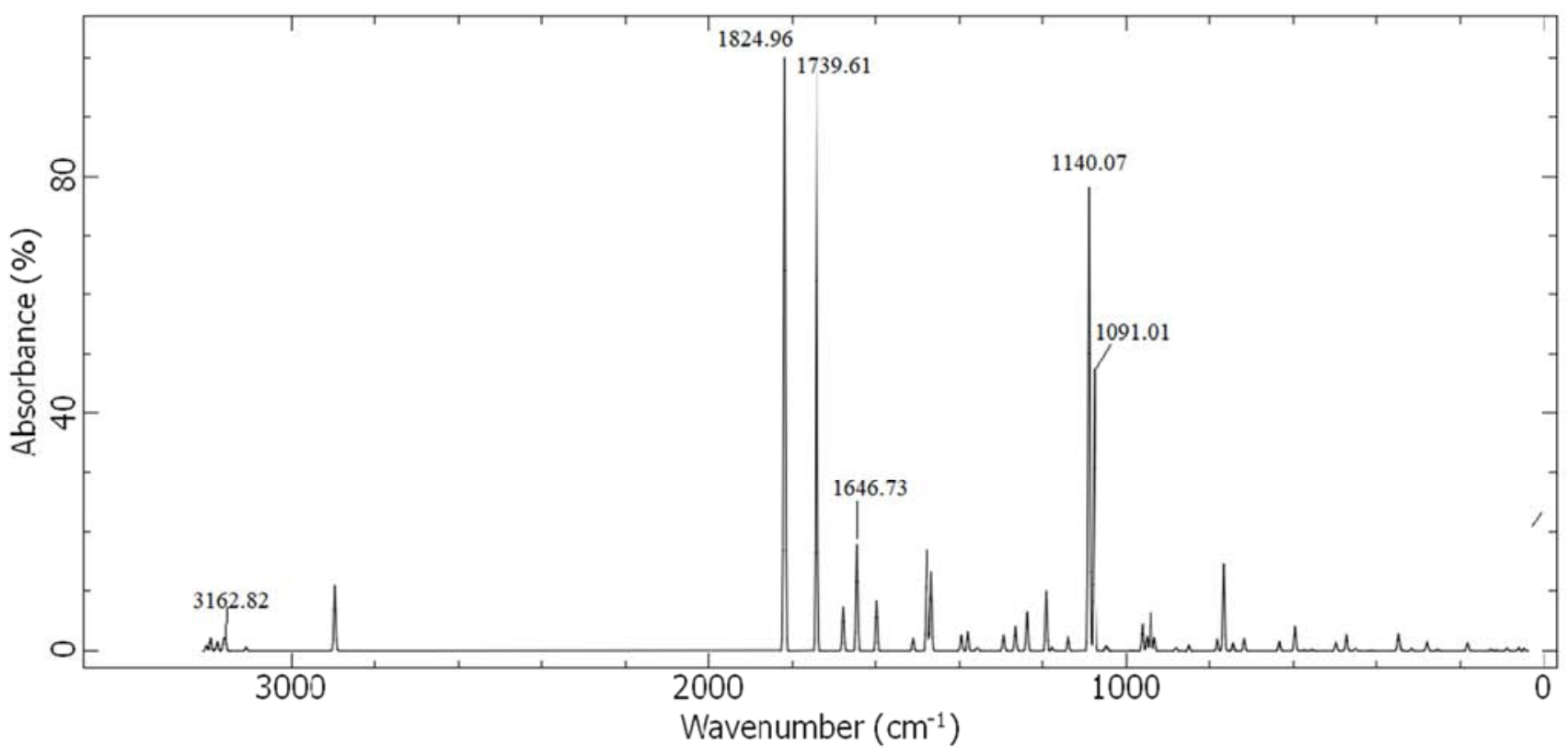

Figure 13. Computed vibrational spectrum.

\subsubsection{Molecular Electrostatic Potential (MEP)}

The molecular electrostatic potential (MEP) is an electrostatic function derived from Coulomb forces that describes the interactions between electrons and the nuclear charges. It plays important role in inspecting reactivity since an approaching electrophile will be attracted into negative sites.

It is defined in atomic units, a.u, as follows [23, 24]:

$$
V(r)=\sum_{A} \frac{Z_{A}}{\left|R_{A}-r\right|}-\int \frac{\rho\left(r^{\prime}\right)}{\left|r^{\prime}-r\right|} d^{3} r^{\prime}
$$

where $Z_{A}$ is the charge of nucleus A sited at $R_{A}, \rho\left(r^{\prime}\right)$ is the electronic density function of the molecule, and $r^{\prime}$ is the dummy integration variable.

In this study, we have calculated MEP with optimized geometries from DFT / RB3LYP, RHF, FREQ / RB3LYP and B3PW91 methods utilizing the $6-311^{++} \mathrm{G}(\mathrm{d}, \mathrm{p})$ basis set to supply information with respect to these reactive sites to distinguish the electrophilic and nucleophilic areas. The outcomes of these calculations are presented in color visualizations (Figure 14). In this figure, the red color in the figure indicates areas of higher negative potential which are favorable for electrophilic attack, while the blue color (higher positive potential) identifies beneficial sites for nucleophilic attack. In Figure 14, two possible areas on compound (I) for electrophilic attack are discernible indicating negative sites. These regions are situated on $\mathrm{O} 2$ and around $\mathrm{O} 3$ and $\mathrm{O} 4$ oxygen atoms with maximum values of $-0.0552,-0.0583$, 0.0608 and -0.0606 a.u. for DFT/RB3LYP, FREQ/RB3LYP, RHF and B3PW91 methods, respectively. Therefore, Figure 14 corroborates the presence of the intermolecular C11- 
H11C...O3 interaction.
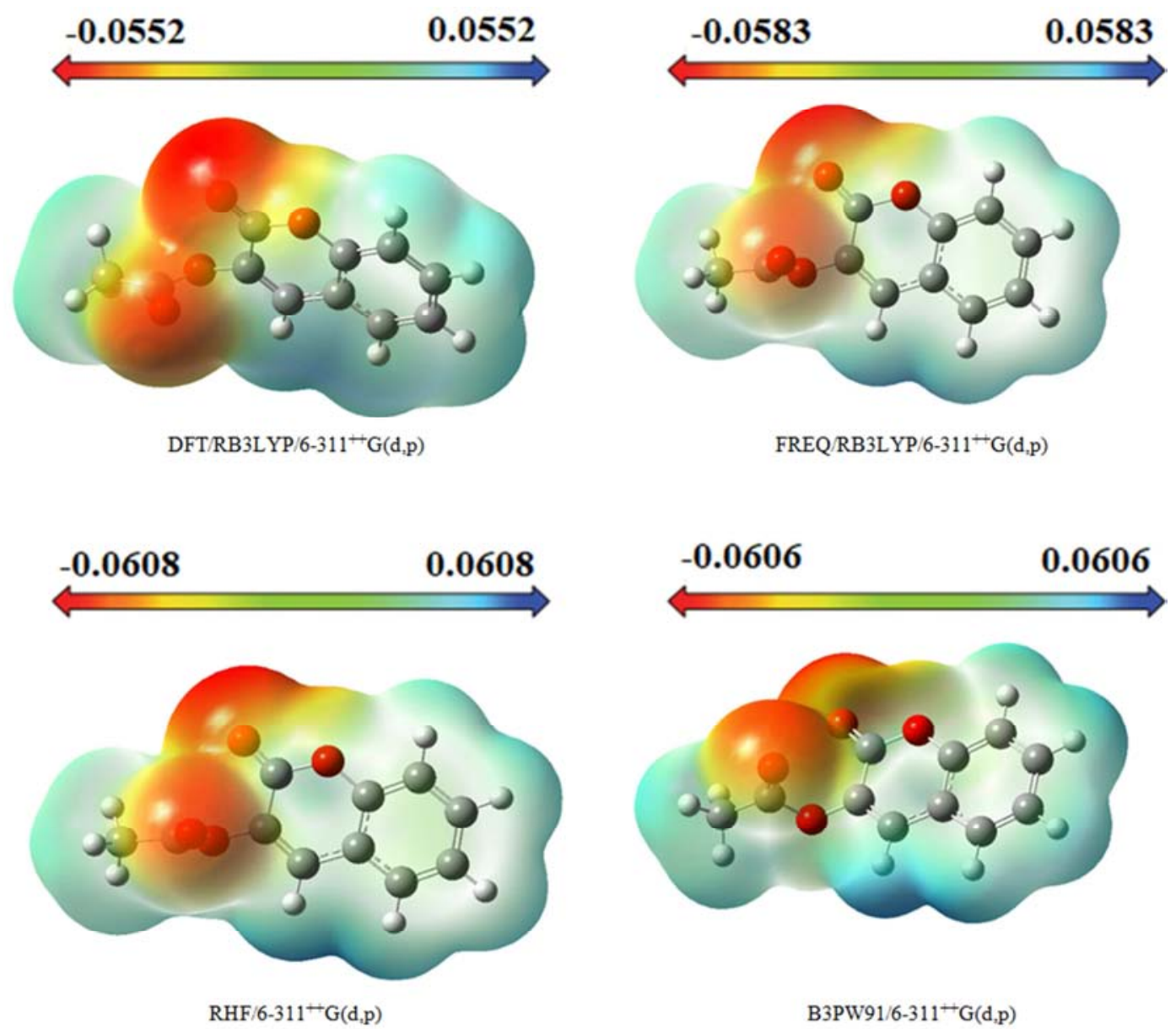

Figure 14. Molecular electrostatic potential map (MEP) (in a.u.) calculated at DFT/RB3LYP, FREQ/RB3LYP, RHF and B3PW91 level frontier molecular orbitals analysis with $6-311^{++} G(d, p)$ basis set.

\subsubsection{HOMO-LUMO Analysis}

The outcomes of the apportionments and energy levels of the topmost occupied molecular orbital (HOMO) and the unoccupied molecular orbital (LUMO) calculated using four quantum chemical methods are depicted in figure 15. The computations disport that compound (I) possesses 53 occupied molecular orbitals and the energy gap between the LUMO and HOMO levels are 4.574 (DFT/B3LYP), 4.627 (FREQ/ B3LYP), 4.606 (B3PW91) and $9.858 \mathrm{eV}$ (RHF), successively. The first three computational results of HOMOLUMO gaps (DFT/B3LYP, FREQ/ B3LYP, B3PW91 methods) in the range 4.574-4.627 eV demonstrate that 2oxo-2H-chromen-3-yl acetate is polarizable together with a high chemical reactivity and low kinetic stability and is also called soft molecule [25]. The latter value $(\Delta E=9.858 \mathrm{eV})$, almost double the previous ones could suggest that RHF method is not suitable for estimating energy gap between the LUMO and HOMO levels. The HOMO and LUMO energies, the energy gap $(\Delta E)$, the ionization potential $(I)$, the electron affinity $(A)$, the absolute electronegativity $(\chi)$, the absolute hardness $(\eta)$, the softness $(S)$ and the optimization energy (E) for the studied compound have been estimated and the outcomes are displayed in Table 8. By utiltzing HOMO and
LUMO energy values for a molecule, electronegativity and chemical hardness can be expressed as follows [26]:

$$
\begin{gathered}
\chi=-\left(\mathrm{E}_{\mathrm{HOMO}}+\mathrm{E}_{\mathrm{LUMO}}\right) / 2 \\
\eta=\left(\mathrm{E}_{\mathrm{LUMO}}-\mathrm{E}_{\mathrm{HOMO}}\right) / 2 \\
S=1 / 2 \eta \\
I=-E_{\text {Hомо }} \\
A=-E_{\text {LUMO }} . \\
\Delta E=\mathrm{E}_{\text {LUMO }}-\mathrm{E}_{\text {Hомо }}
\end{gathered}
$$

Table 8. The computed chemical properties of (I) using $6-311^{++} G(d, p)$ basis set.

\begin{tabular}{lllll}
\hline & DFT/ B3LYP & FREQ/ B3LYP & RHF & B3PW91 \\
\hline $\mathrm{E}_{\text {LUмо }}(\mathrm{eV})$ & -2.305 & -2.277 & 0.855 & -2.303 \\
$\mathrm{E}_{\text {номо }}(\mathrm{eV})$ & -6.879 & -6.904 & -9.003 & -6.909 \\
$\mathrm{I}(\mathrm{eV})$ & 6.8879 & 6.904 & 9.003 & 6.909 \\
$\mathrm{~A}(\mathrm{eV})$ & 2.305 & 2.277 & -0.855 & 2.303 \\
$\chi(\mathrm{eV})$ & 4.59 & 4.59 & 4.07 & 4.61 \\
$\eta(\mathrm{eV})$ & 2.29 & 2.31 & 4.93 & 2.30 \\
$\left.S(\mathrm{eV})^{-1}\right)$ & 0.22 & 0.22 & 0.10 & 0.22 \\
$\Delta \mathrm{E}(\mathrm{eV})$ & 4.574 & 4.627 & 9.858 & 4.606 \\
$\mathrm{E}(\mathrm{au})$ & -725.10 & -724.95 & -720.86 & -724.81 \\
\hline
\end{tabular}



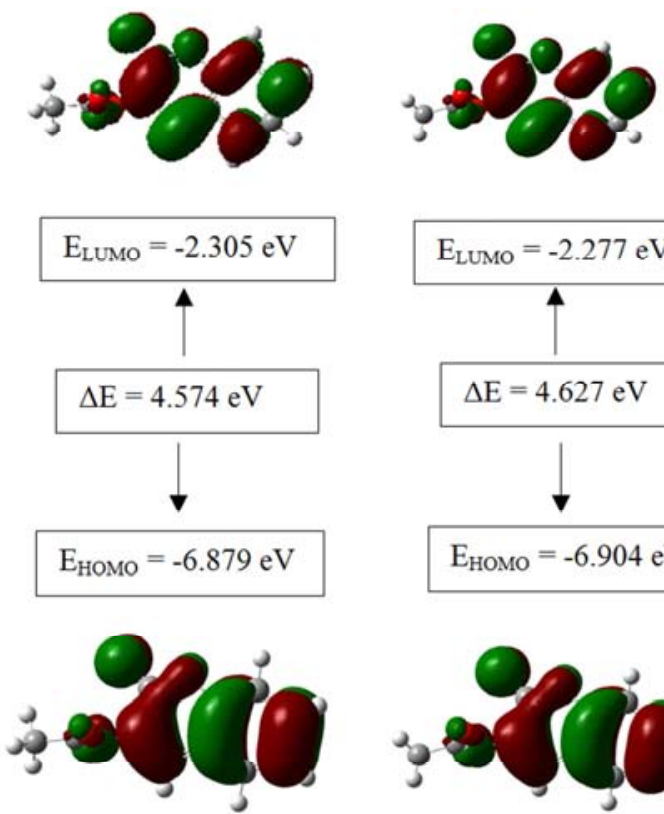

DFT/RB3LYP/6-311 + G(d,p)
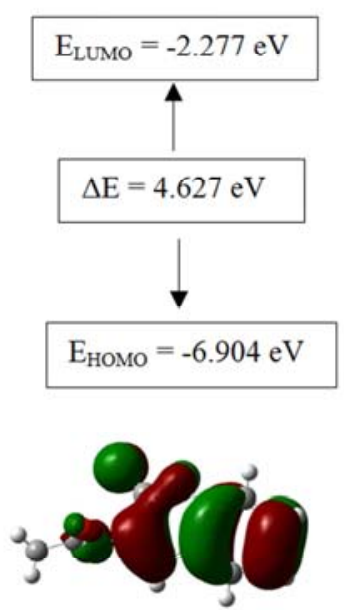

FREQ/RB3LYP/6-311 + G(d,p)
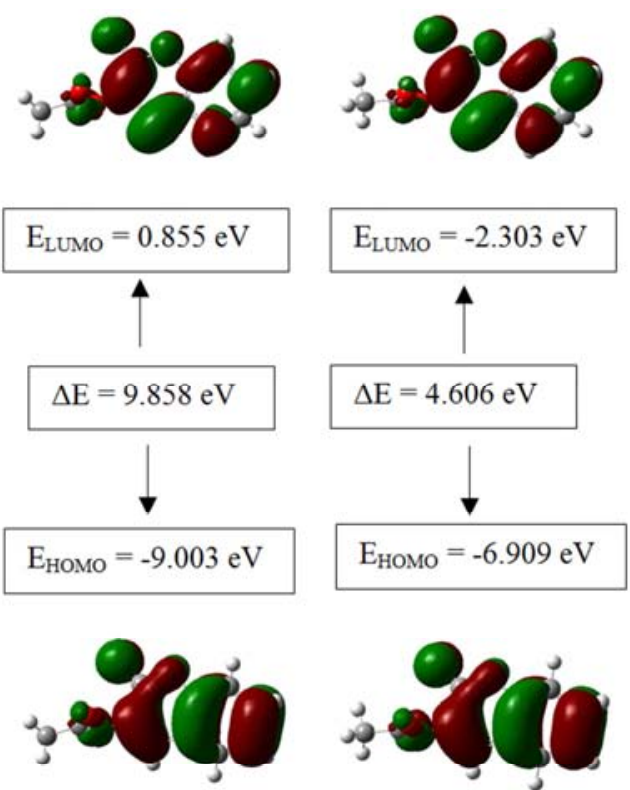

RHF $/ 6-311^{++} \mathrm{G}(\mathrm{d}, \mathrm{p})$

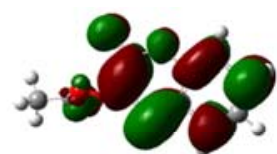

B3PW91/6-311 ${ }^{++} \mathrm{G}(\mathrm{d}, \mathrm{p})$

Figure 15. The distributions and energy levels of the HOMO and LUMO orbitals computed for compound (I).

Table 9. Computed Mulliken atomic charges utilizing $6-311^{++} G(d, p)$ basis set.

\begin{tabular}{lllll}
\hline Atom & DFT/RB3LYP & FREQ/RB3LYP & RHF & B3PW91 \\
\hline O1 & -0.107 & -0.123 & -0.272 & -0.054 \\
O2 & -0.271 & -0.278 & -0.366 & -0.260 \\
O3 & -0.016 & -0.029 & -0.103 & -0.039 \\
O4 & -0.196 & -0.207 & -0.281 & -0.178 \\
C1 & 0.616 & 0.585 & 0.912 & 0.730 \\
C2 & -0.559 & -0.718 & -862 & -0.876 \\
C3 & 0.165 & 0.517 & 0.752 & 0.727 \\
H3 & 0.199 & 0.170 & 0.248 & 0.238 \\
C4 & 2.32 & 1.934 & 1.543 & 1.990 \\
C5 & -1.961 & -1.905 & -2.088 & -2.363 \\
C6 & -0.407 & -0.367 & -0.187 & 0.210 \\
H6 & 0.210 & 0.182 & 0.245 & 0.177 \\
C7 & -0.348 & -0.253 & -0.364 & -0.251 \\
H7 & 0.175 & 0.141 & 0.215 & 0.209 \\
C8 & -0.137 & -0.048 & -0340 & -0.409 \\
H8 & 0.174 & 0.140 & 0.203 & 0.216 \\
C9 & -0.112 & 0.018 & 0.330 & -0.450 \\
H9 & 0.140 & 0.105 & 0.185 & 0.246 \\
C10 & 0.084 & 0.041 & 0.238 & 0.068 \\
C11 & -0.510 & -0.354 & -0.543 & -0.619 \\
H11A & 0.174 & 0.147 & 0.167 & 0.190 \\
H11B & 0.169 & 0.150 & 0.171 & 0.195 \\
H11C & 0.199 & 0.153 & 0.197 & 0.226 \\
\hline & & & & \\
& & & & \\
\hline
\end{tabular}

\subsubsection{The Mulliken Charge Population}

Mulliken charges originate from the Mulliken population examination and give a means of evaluating partial nuclear charges from calculations carried out by the methods of computational chemistry. These atomic charges affect a lot of properties of molecular systems such as dipole moment, molecular polarizability, electronic structure, and so on. The charge distributions calculated by the Mulliken method [27-30] for optimized geometry of compound (I) is summarized in table
9. The calculated charge of $\mathrm{O} 2$ oxygen atom is negative and that of the carbon atom $\mathrm{C} 10$ is positive for all methods used (table 9). The resulting electrostatic interactions between them are materialized by the short intramolecular $\mathrm{C} 10 \ldots \mathrm{O} 2$ bond in the solid state (figure 8). Further, the charges of the three atoms $(\mathrm{C} 11 ; \mathrm{H} 11 \mathrm{C} ; \mathrm{O} 3)$ extracted from table 9 are $(-0.510 \mathrm{e} ; 0.199 \mathrm{e}$;0.016 e), (-0.354 e; 0.153 e; -0.029 e), (-0.543e; 0.197 e; -0.103 e) and (-0.619 e; $0.226 \mathrm{e} ;-0.039$ e) for the DFT/RB3LYP/6$311^{++} \mathrm{G}(\mathrm{d}, \mathrm{p})$, FREQ/RB3LYP/6-311 ${ }^{++} \mathrm{G}(\mathrm{d}, \mathrm{p})$, RHF/6-311 ${ }^{++} \mathrm{G}(\mathrm{d}, \mathrm{p})$ and B3PW91/6-311 ${ }^{++} \mathrm{G}(\mathrm{d}, \mathrm{p})$ methods, respectively. These values highlighting interactions between $\mathrm{H} 11 \mathrm{C}$ and $\mathrm{O} 3$ atoms confirm the intermolecular hydrogen bond $\mathrm{C} 11-\mathrm{H} 11 \mathrm{C} \ldots \mathrm{O} 3[-x+1 / 2$, $y+1 / 2, z]$. Likewise, the computed Mulliken charges of $\mathrm{C} 7$ and C8 are negatives pointing out that these carbon atoms are electronegatives. Thus, they can establish other hydrogen bonds in the gaseous state.

\subsubsection{Non-linear Optical Properties}

Since the discovery of nonlinear optical (NLO) phenomenon for the first time in 1961, nonlinear optical frequency conversion materials have played major role in many fields, such as optical communication, laser technology, optical data storage, optical information processing, image manipulation etc [31 32]. The very first material used to exploit for their nonlinear optical (NLO) properties was potassium dihydrogen phosphate (KDP). It was used in the first experiments in nonlinear optics and is still used widely in nonlinear optics devices.

Currently, Urea is used to replace KDP due to its second harmonic efficiency which is three times higher than that of the KDP crystals [33].

At present, because of potential applications in various fields, a huge number of investigate papers in new materials 
indicating effective nonlinear optical properties have been of awesome interest [34-38]. So, the use of quantum chemical methods such as density functional theory (DFT), HartreeFock (HF) and B3PW91 for molecular hyperpolarizabilities is awaited to supply a supervision and accelerate upcoming experimental studies [39, 40].

Hence, within the display job, we have minimized the geometries with GAUSSIAN-09W program package [16] at DFT / RB3LYP, RHF and B3PW91methods using 6-311 ${ }^{++} \mathrm{G}(\mathrm{d}$, p) basis set and computed the dipole moments $(\mu)$, the polarizabilities $(<\alpha>)$, the anisotropy of the polarizabilities $(<\Delta \alpha>)$ and the first-order hyperpolarizabilities $(\beta)$ which gives information about the material capability to generate second order non-linear effects [41]. These parameters are defined as [42-43]:

$$
\begin{gathered}
\mu=\left(\mu_{x}^{2}+\mu_{y}^{2}+\mu_{z}^{2}\right)^{\frac{1}{2}} \\
\langle\alpha\rangle=\frac{\alpha_{x x}+\alpha_{y y}+\alpha_{z z}}{3} \\
\langle\Delta \alpha\rangle=\left[\frac{\left(\alpha_{x x}-\alpha_{y y}\right)^{2}+\left(\alpha_{y y}-\alpha_{z z}\right)^{2}+\left(\alpha_{z z}-\alpha_{x x}\right)^{2}+6\left(\alpha_{x y}^{2}+\alpha_{x z}^{2}+\alpha_{y z}^{2}\right)}{2}\right]^{\frac{1}{2}} \\
\beta=\left[\left(\beta_{x x x}+\beta_{x y y}+\beta_{x z z}\right)^{2}+\left(\beta_{y y y}+\beta_{y z z}+\beta_{y x x}\right)^{2}+\right. \\
\left.\left(\beta_{z z z}+\beta_{z x x}+\beta_{z y y}\right)^{2}\right]^{\frac{1}{2}}
\end{gathered}
$$

All the numerical outcomes of the tensors regarding the polarizability were expressed to electronic units (esu) and recorded in Tables 10, 11 and 12, with $\left(\alpha: 1\right.$ a.u. $=0.1482 \times 10^{-}$ ${ }^{24} \mathrm{esu} ; \beta$ : 1 a.u. $\left.=8.6393 \times 10^{-33} \mathrm{esu}\right)[44]$.

Table 10. Computed dipole moment (D) using the $6-311^{++} G(d, p)$ basis set.

\begin{tabular}{|c|c|c|c|c|c|c|c|c|c|c|c|}
\hline & $\begin{array}{l}\beta_{\mathrm{xxx}} \\
\text { (a.u) }\end{array}$ & $\begin{array}{l}\beta_{\text {yyy }} \\
\text { (a.u) }\end{array}$ & $\begin{array}{l}\beta_{\mathrm{zzz}} \\
\text { (a.u) }\end{array}$ & $\begin{array}{l}\beta_{\text {xyy }} \\
\text { (a.u) }\end{array}$ & $\begin{array}{l}\beta_{\mathrm{xxy}} \\
\text { (a.u) }\end{array}$ & $\begin{array}{l}\beta_{\mathrm{xxz}} \\
\text { (a.u) }\end{array}$ & $\begin{array}{l}\beta_{\mathrm{xzz}} \\
\text { (a.u) }\end{array}$ & $\begin{array}{l}\beta_{\text {yzz }} \\
\text { (a.u) }\end{array}$ & $\begin{array}{l}\beta_{\text {yyz }} \\
\text { (a.u) }\end{array}$ & $\begin{array}{l}\beta_{\text {xyz }} \\
\text { (a.u) }\end{array}$ & $\begin{array}{l}\beta \times 10^{-30} \\
(\mathrm{esu})\end{array}$ \\
\hline DFT/RB3LYP & $-35,02$ & $-21,93$ & $-6,21$ & 11,07 & $-10,02$ & $-4,71$ & $-5,49$ & 9,91 & 1,59 & 0,65 & 0.3278 \\
\hline RHF & $-36,10$ & $-24,70$ & $-6,586$ & 11,24 & $-9,55$ & $-4,30$ & $-6,43$ & 8,10 & 0,75 & 0,07 & 0.3630 \\
\hline
\end{tabular}

\begin{tabular}{lllr}
\hline & $\boldsymbol{\mu}_{\mathbf{x}}$ & $\boldsymbol{\mu}_{\mathbf{y}}$ & \multicolumn{1}{c}{$\boldsymbol{\mu}_{\mathbf{z}}$} \\
\hline DFT/RB3LYP & 2.2716 & -3.8227 & -1.4063 \\
RHF & 2.3609 & -4.0474 & -1.6281 \\
B3PW91 & 2.2120 & -3.7658 & -1.3740 \\
\hline
\end{tabular}

Table 11. All $\alpha \times 10^{-24}$ (esu) components, $<\alpha>\times 10^{-24}$ (esu) and $<\Delta \alpha>\times 10^{-24}$ (esu) values computed with the 6-311 ${ }^{++}$G(d,p) basis set.

\begin{tabular}{llllllll}
\hline & $\boldsymbol{\alpha}_{\mathbf{x x}}$ & $\boldsymbol{\alpha}_{\mathbf{x y}}$ & $\boldsymbol{\alpha}_{\mathbf{y y}}$ & $\boldsymbol{\alpha}_{\mathbf{x z}}$ & $\boldsymbol{\alpha}_{\mathbf{y z}}$ & $\boldsymbol{\alpha}_{\mathbf{z z}}$ & $<\boldsymbol{\alpha}>$ \\
\hline DFT/RB3LYP & 9.81 & 0.80 & 12.71 & 0.80 & 0.28 & 13.74 & 12.09 \\
RHF & $-9,80$ & 0,81 & $-12,72$ & 0,87 & 0,36 & $-13,88$ \\
B3PW91 & 9,65 & 0,8 & 12,51 & 0,74 & 0,28 & 12.07 & 13,62 \\
\hline
\end{tabular}

Table 12. First-order hyperpolarizabilities ( $\beta$ ) values of (I) computed with DFT, HF and B3PW91 methods.

For investigation, urea molecule with its parameters $\left(<\mu \quad>=1.37 \mathrm{D}, \quad<\alpha>=3.83 \times 10^{-24}\right.$ esu $[45-47], \quad$ and $<\beta>=0.1947 \times 10^{-30}$ esu [48]) is utilized for comparison in the study of NLO properties of compound (I). As can be famous from tables (10-12), the values of all parameters for (I) are somewhat higher than those of the urea molecule.

For additional subtleties, the dipole moments of compound (I) originating from the DFT / B3LYP, HF and B3PW91 calculations are 3.40, 3.62 and 3.34 times higher than that of the urea molecule, respectively (Table 10). Similarly, the calculated first-order hyperpolarizabilities are also 1.68, 1.86 and 1.73 times higher than that of the urea molecule. In addition, the estimated values of the average linear polarizability $\langle\alpha\rangle$ are close to that of the urea molecule, i.e. 1.06 (DFT / B3LYP), 1.09 (HF) and 1.06 (B3PW91) (table 11).

These values demonstrate a favorable first order hyperpolarizability of 2-oxo-2H-chromen-3-yl acetate which can generate second order non-linear effects. Therefore, 2oxo-2H-chromen-3-yl acetate can be considered a potential candidate having nonlinear optical effects and may have potential applications in the development of NLO materials.

\section{Conclusion}

In ouline, we began our study with the synthesis of the structure and characterized it by spectrometry and by X-ray crystallography. Also, we have inspected its intermolecular interactions by means of Hirshfeld surface analysis.

Then the estimated geometric parameters and infrared spectrum originating from quantum chemical calculations are compared respectively with the $\mathrm{x}$-ray crystal determination data and the experimental IR spectrum. In this comparison, we can notice that there are no notable differences except the experimental torsion angle, $\mathrm{C} 2$ $\mathrm{O} 3-\mathrm{C} 10-\mathrm{O} 4$, which differs from the calculated values by around $12^{\circ}$. Moreover, molecular electrostatic potential, HOMO-LUMO examination and the Mulliken charge population of 2-oxo-2H-chromen-3-yl acetate have been explored utilizing DFT/RB3LYP, FREQ/RB3LYP, HF and B3PW91 calculations outcomes. The generated MEP maps indicate the areas of negative potential which are located around electronegative atoms i.e oxygen atoms and the positive potential which is located around the hydrogen 
atoms. These regions highlight the areas from where the compound can establish intra- and intermolecular interactions.

Additionally, Mulliken charges computed in the gaseous state show partial negative charges on atoms $\mathrm{O} 2, \mathrm{O} 3, \mathrm{C} 11$ and partial positive charges on $\mathrm{H} 11 \mathrm{C}, \mathrm{C} 10$ confirming the intermolecular hydrogen bond $\mathrm{C} 11-\mathrm{H} 11 \mathrm{C} \ldots \mathrm{O} 3[-x+1 / 2$, $y+1 / 2, z]$ and the short intramolecular $\mathrm{C} 10 \ldots \mathrm{O} 2$ bond.

Endly, the calculated values of the first-order hyperpolarizabilities $(\beta)$ with DFT/B3LYP, HF and B3PW91 methods have suggested non-linear optical properties of the studied compound.

\section{Supplementary Materials}

CCDC-2052679 contains the supplementary crystallographic data for this paper. These data can be obtained free of charge via https://www.ccdc.cam.ac.uk/structures/, or by e-mailing data_request@ccdc.cam.ac.uk, or by contacting The Cambridge Crystallographic Data Centre, 12 Union Road, Cambridge CB2 1EZ, UK; fax: +44(0)1223-336033.

\section{Conflicts of Interest}

The authors declare that they have no conflict of interest.

\section{Acknowledgements}

The authors offer their thanks to the Spectropole Service of the Federation of Chemical Sciences (Aix-Marseille University, France) for the spectroscopic investigation.

\section{References}

[1] Yu, D.; Suzuki, M.; Xie, L.; Morris-Natschke, S. L. \& Lee, K.-H.; (2003) Med. Res. Rev. 23, 322-345.

[2] Yu, D.; Morris-Natschke, S. L. \& Lee, K.-H.; (2007) Med. Res. Rev. 27, 108-132.

[3] Abernethy, J. L.; (1969). J. Chem. Educ. 46, 561-568.

[4] Vukovic, N.; Sukdolak, S.; Solujic, S. \& Niciforovic, N.; (2010) Arch. Pharm. Res. 33, 5-15.

[5] Wang, M.; Wang, L.; Li, Y. \& Li, Q.; (2001) Transition Met. Chem. 26, 307-310.

[6] Basanagouda, M.; Kulkarni, M. V.; Sharma, D.; Gupta, V. K.; Pranesha, P.; Sandhyarani, P. and Rasal V. P.; (2009) J. Chem. Sci., 121, 485-495.

[7] Emmanuel-Giota, A. A.; Fylaktakidou, K. C.; Litinas, K. E.; Nicolaides, D. N. and Hadjipavlou-Litina, D. J.; (2001) Heterocycl. Chem., 38, 717-722.

[8] Abou, A.; Djandé, A.; Kakou-Yao, R.; Saba, A. and Tenon, A. J.; (2013) Acta Cryst., E69, o1081-o1082.

[9] Ouédraogo, M.; Abou, A.; Djandé, A.; Ouari, O. and Zoueu, T. J.; (2018) Acta Cryst., E74, 530-534.
[10] Rigaku, OD.; (2015) CrysAlis PRO. Rigaku Oxford Diffraction, Yarnton, England.

[11] Burla, M. C.; Caliandro, R.; Carrozzini, B.; Cascarano, G. L.; Cuocci, C.; Giacovazzo, C.; Mallamo, M.; Mazzone, A. and Polidori, G., J.; (2015) Appl. Cryst., 48, 306-309.

[12] Farrugia, L. J.; (2012) J. Appl. Cryst., 45, 849-854.

[13] Sheldrick, G. M.; (2015) Acta Cryst., C71, 3-8.

[14] Spek, A. L.; (2009) Acta Cryst., D65, 148-155.

[15] Wolff, S. K.; Grimwood, D. J.; McKinnon, J. J.; Turner, M. J.; Jayatilaka, D. and Spackman, M. A.; (2012) Crystal Explorer. The University of Western Australia.

[16] Frisch, M. J.; Trucks, G. W.; Schlegel, H. B.; Scuseria, G. E.; Robb, M. A.; Cheeseman, J. R.; et al.; (2013) GAUSSIAN09. Gaussian, Inc., Wallingford, CT, USA.

[17] Allen, F. H.; Walson, D. G.; Orpen, A. G.; Taylor, R.; (1987) J. Chem. Soc., Perkin Trans. II, S1-S9.

[18] Gomes, L. R.; Low, J. N.; Fonseca, A.; Matos, M. J. and Borges, F.; (2016) Acta Cryst., E72, 926-932.

[19] Ziki, E.; Yoda, J.; Djandé, A.; Saba, A. and Kakou-Yao, R.; (2016) Acta Cryst., E72, 1562-1564.

[20] Cremer, D. \& Pople, J.; (1975). J. Am. Chem. Soc. 97, 13541358.

[21] Bitzer, S. R.; Visentin, C. L.; Hörner, M.; Nascimento, M. A. C. and Filgueiras, C. A. L.; (2017) J. Mol. Struct., 1130, 165-173.

[22] Hanwell, M. D.; Curtis, D. E.; Lonie, D. C.; Vandermeersch, T.; Zurek, E. and Hutchison, G. R.; (2012) Journal of Cheminformatics, 4 (1), 17.

[23] Murray, J. S.; Politzer, P.; The electrostatic potential: an overview. WIREs Comp Mol Sci, 2011, 1: 153-163

[24] Politzer, P. and Murray, J. S.; (2002) Theoretical Chemistry Accounts, 108 (3), 134-142.

[25] Fleming, I.; (1976) Frontier Orbitals and Organic Chemical Reactions, John Wiley \& Sons, London, UK.

[26] Pearson, R. G.; (1986) Proceedings of the National Academy of Sciences of the United States of America, 83 (22), 84408841 .

[27] Mulliken, R. S.; (1955) The Journal of Chemical Physics, 23 (10), 1833-1840.

[28] Mulliken, R. S.; (1955) The Journal of Chemical Physics, 23 (10), 1841-1846.

[29] Mulliken, R. S.; (1955) The Journal of Chemical Physics, 23 (12), 2338-2342.

[30] Mulliken, R. S.; (1955 The Journal of Chemical Physics, 23 (12), 2343-2346

[31] Janarthanan, S.; Samuel, R. S.; Rajan, Y. C.; Pandi, S.; J. Therm. Anal. Calorim., 2012, 107, 1213-1217.

[32] Bhagavannarayana, G.; Riscob, B.; Mohd, S.; Mater. Chem. Phys., 2011, 126, 20-23.

[33] Gomes, E. D.; Venkatraman, V.; Nogueira, E.; Belsley, M.; Garrido, S. P.; Mater Syn., 2000, 115, 225-228). 
[34] Zhang, R.; Du, B.; Sun, G. and Sun, Y.; (2010) Spectrochimica Acta A, vol. 75, no. 3, pp. 1115-1124.

[35] Yazıc1, S.; Albayrak,.; Gümrükçüolu, I.; enel, I. and Büyükgüngör, O.; (2011) Journal of Molecular Structure, vol. 985, no. 2-3, pp. 292-298.

[36] Chemia, D. S. and Zyss, J.; Non Linear Optical Properties of Organic Molecules and Crystal, Academic Press, New York, NY, USA, 1987.

[37] Zyss, J.; Molecular Non Linear Optics, Academic Press, Boston, Mass, USA, 1994.

[38] Ben Ahmed, A.; Feki, H.; Abid, Y. and Minot, C.; (2010) Spectrochimica Acta A, vol. 75, no. 4, pp. 1315-1320.

[39] Suponitsky, K. Y.; Tafur, S; Masunov, A. E.; (2008) Journal of Chemical Physics, 129, 044109-11.

[40] Avci, D.; Başoğlu, A.; Atalay, Y.; (2011) International Journal of Quantum Chemistry, 111, 1, 130-147.

[41] Machado, A. E. H.; Neto, N. M. B.; Ueno, L. T.; et al.; (2008) Journal of Photochemistry Photobiology A-Chemistry, 199, 1,
$23-33$.

[42] Abraham, J. P.; Sajan, D.; Hubert, Joe I. H. and Jayakumar, V. S.; (2008) Spectrochimica Acta Part A, 71, 2, 355-367.

[43] Karamanis, P.; Pouchan, C. and Maroulis, G.; (2008) Physical Review A, 77, 013201-013208.

[44] Ben Ahmed, A.; Feki, H.; Abid, Y.; Boughzala, H. and Mlayah, A.; (2008) Journal of Molecular Structure, 888, 1-3, 180-186.

[45] Nkungli, N. K.; Ghogomu, J. N.; (2016) J. Theor. Chem., 2016, $1-19$.

[46] Pluta, T.; Sadlej, A. J.; (2001) J. Chem. Phys., 114, 136.

[47] Song, X.; Farwell, S. O.; (2004) J. Anal. Appl. Pyrolysis, 71, 901-915.

[48] Eme, A.; Günedodu Sadinç, S.; (2014) BAய̈ Fen Bil. Enst. Dergisi Cilt 16 (1) 47-75. 